\title{
Pacific
}

Journal of

Mathematics

\section{ON THE MULTIPLICITY OF NON-ITERATED PERIODIC BILLIARD TRAJECTORIES}

MARCo MAZZUCCHELLI 


\title{
ON THE MULTIPLICITY OF NON-ITERATED PERIODIC BILLIARD TRAJECTORIES
}

\author{
MARCo MaZZUCCHELLI
}

\begin{abstract}
We introduce an iteration theory for periodic billiard trajectories in a compact and convex domain of the Euclidean space, and we apply it to establish a multiplicity result for non-iterated trajectories.
\end{abstract}

1. Introduction 181

2. Preliminaries 184

3. Iteration theory for periodic billiard trajectories 187

4. Proof of the main result 200

Acknowledgments 204

References 204

\section{Introduction}

Billiard dynamics describes the motion of a particle moving without friction in a compact domain of $\mathbb{R}^{N+1}$, for $N \geq 1$, while being subject to a singular potential which is identically zero in the interior of the domain and $+\infty$ on the boundary. This implies that the particle moves on straight lines with constant speed until it reaches the boundary of the domain, where it bounces with specular reflection and with no loss of energy. In this paper, we investigate the multiplicity of certain periodic billiard trajectories in strictly convex domains of $\mathbb{R}^{N+1}$ enclosed by a smooth hypersurface, which is therefore diffeomorphic to the unit sphere $S^{N}$. Throughout the paper, by strict convexity we will always mean that the second fundamental form of the boundary is everywhere positive definite.

Historically, the first multiplicity result for periodic billiard trajectories was proved by Birkhoff [1927] for convex plane billiards $(N=1)$. The result asserts that, for each pair of coprime positive integers $n \geq 2$ and $r \leq n / 2$, there are at least two distinct periodic billiard trajectories with $n$ bounce points and rotation number $r$. The plane case is special, since the billiard dynamics can be described by means of an area-preserving twist map on the annulus, and nowadays Birkhoff's Theorem can be proved by means of Aubry-Mather theory; see [Bangert 1988]

MSC2010: 37J45, 55R80.

Keywords: billiards, Morse theory, iteration of periodic trajectories. 
and references therein. For higher-dimensional billiards the problem is essentially harder; estimates for the number of bounce trajectories with prescribed number of bounce points have been proved by Farber and Tabachnikov [2002a; 2002b], who corrected and extended an earlier proof by Babenko [1990]. Further extensions, together with more sophisticated multiplicity results, have been proved in [Farber 2002; Karasev 2009]. Roughly, these results can be summarized by saying that, for each $n$ odd, the number of periodic billiard trajectories whose number of bounce points divides $n$ grows at least linearly in $n$.

The arguments in these papers are based on critical point theory. In fact, billiard trajectories can be characterized by a variational principle, which in the periodic case goes as follows. If $S$ is the smooth boundary of our convex billiard table and $n \in \mathbb{N}$, the length functional $L^{\times n}$, defined on the $n$-fold cross product of $S$, computes the perimeter of the $n$-gon inscribed by a given sequence of points $\boldsymbol{q}=$ $\left(q_{0}, \ldots, q_{n-1}\right) \in S^{\times n}$. This functional is clearly continuous, and actually smooth when restricted to the so-called cyclic configuration space $\mathrm{G}^{\times n}(S)$, the space of those $\boldsymbol{q}$ 's such that $q_{j} \neq q_{j+1}$ for all $j \in \mathbb{Z}_{n}$. The critical points $\boldsymbol{q} \in \mathrm{G}^{\times n}(S)$ of $L^{\times n}$ are precisely the $n$-periodic sequences of bounce points of billiard trajectories. Notice that the dihedral group $\mathbb{D}_{n}$ acts by permutations on the cyclic configuration space $\mathrm{G}^{\times n}(S)$, and all the points that belong to a same orbit of its action refer to the same geometric closed curve.

Now, the lack of compactness of $\mathrm{G}^{\times n}(S)$ does not represent a real obstacle for applying the machinery of critical point theory with the length functional $L^{\times n}$ or, more precisely, with the functional $-L^{\times n}-$ see [Farber and Tabachnikov 2002b, Section 4] or the paragraph after Proposition 2.1 below. In particular, each $\mathbb{D}_{n}$ equivariant homology or cohomology class of $\mathrm{G}^{\times n}(S)$ gives rise to a periodic billiard trajectory (as critical point of $L^{\times n}$ ) and, at least in the nondegenerate case, linearly independent classes produce different critical points. In [Farber and Tabachnikov 2002b], the equivariant cohomology algebra $\mathrm{H}_{\mathbb{D}_{n}}^{*}\left(\mathrm{G}^{\times n}(S) ; \mathbb{Z}_{2}\right)$ has been computed for each $n$ odd. Its rank gives, in the nondegenerate case, a lower bound for the number of periodic billiard trajectories whose number of bounce points divides $n$. In the degenerate case, by Lyusternik-Schnirelmann theory, such a lower bound is given by the cup-length of $\mathrm{H}_{\mathbb{\mathbb { D }}_{n}}^{*}\left(\mathrm{G}^{\times n}(S) ; \mathbb{Z}_{2}\right)$ plus one.

In this paper, we proceed along a different line: the result we prove concerns the multiplicity of periodic orbits whose number of bounce points lies in the set of powers of a given prime number $p$. Our proof uses a minimum amount of information on the homology of the configuration space: we will only need that, for each $n \in \mathbb{N}$, the homology group $\mathrm{H}_{*}\left(\mathrm{G}^{\times n}(S) ; \mathbb{Z}_{2}\right)$ is nontrivial in degree $N-1$, where $N=\operatorname{dim}(S)$. Via Morse theory, for each $n \in \mathbb{N}$, a nonzero homology class in $\mathrm{H}_{N-1}\left(\mathrm{G}^{\times n}(S) ; \mathbb{Z}_{2}\right)$ generates a periodic billiard trajectory $\gamma_{n}$ whose number of bounce points divides $n$. More precisely, if the number of its bounce points is $n / m$, 
the generated critical point $\boldsymbol{q}_{n}=\left(q_{n, 0}, \ldots, q_{n, n}\right) \in \mathrm{G}^{\times n}(S)$ of $L^{\times n}$ is the sequence of bounce points of the $m$-fold iteration of $\gamma_{n}$. Since we only make use of the homology of $\mathrm{G}^{\times n}(S)$ in degree $N-1$, all the $\gamma_{n}$ 's (i.e., all the associated critical points of the length functionals $L^{\times n}$ ) have Morse coindex less or equal than $N-1$ and are not local maxima for $L^{\times n}$.

The main issue here is to prove that, varying $n$, we obtain infinitely many distinct trajectories $\gamma_{p^{n}}$. In order to prove this, we develop an iteration theory for periodic billiard trajectories, a discrete version of the one for closed geodesics (see [Bott 1956; Gromoll and Meyer 1969b; Bangert and Klingenberg 1983], for example), that may also have independent interest. More specifically, we investigate the behavior of the Morse indices and of the local homology of billiard periodic trajectories under iteration, and we prove a discrete version of Bangert and Klingenberg's homological vanishing under iteration, a tool that can be used in certain situations to assert the existence of infinitely many closed geodesics.

The main result of the paper is the following.

Theorem 1.1. Consider the billiard dynamics in a strictly convex subset enclosed by a smooth hypersurface of $\mathbb{R}^{N+1}$, where $N \geq 2$. For each prime number $p$ at least one of the following two statements is satisfied:

- There is some $n \in \mathbb{N}$ such that infinitely many periodic billiard trajectories bounce $p^{n}$ times.

- There is a sequence $\left\{\gamma_{\alpha} \mid \alpha \in \mathbb{N}\right\}$ of geometrically distinct periodic billiard trajectories such that

(i) each $\gamma_{\alpha}$ bounces $p^{n_{\alpha}}$ times for some $n_{\alpha} \in \mathbb{N}$, and

(ii) each $\gamma_{\alpha}$ has Morse coindex at most $N$ and is not a local maximum for the length functional $L^{\times p^{n_{\alpha}}}$.

We stress that Theorem 1.1 does not follow from the mere knowledge of the cohomology of the cyclic configuration space (and thus, it does not follow from [Farber and Tabachnikov 2002b, Theorem 1]). In fact, the Poincaré polynomial associated to $\mathrm{H}_{\mathbb{D}_{n}}^{*}\left(\mathrm{G}^{\times n}(S) ; \mathbb{Z}_{2}\right)$ is given by

$$
P_{N, n}(t)=\frac{\left(t^{(n-1)(N-1)}-1\right)\left(t^{N}-1\right)\left(t^{N}+1\right)}{\left(t^{2(N-1)}-1\right)(t-1)}=\left(\sum_{j=0}^{(n-3) / 2} t^{2(N-1) j}\right)\left(\sum_{k=0}^{N-1} t^{k}\right)\left(t^{N}+1\right)
$$

provided $N=\operatorname{dim}(S) \geq 3$ and $n$ is odd; see Theorem 7 and proof of Proposition 4.5 in [Farber and Tabachnikov 2002b]. ${ }^{1}$ Even in the nondegenerate case, by $\mathbb{D}_{n}$-equivariant Morse theory, a lower bound for the number of periodic billiard

\footnotetext{
${ }^{1}$ In the theorem cited, the formula of the Poincaré polynomial contains a typo.
} 
trajectories having Morse coindex less or equal than $N$ and number of bounce points that divides $n$ is only given by

$$
\begin{aligned}
\sum_{j=0}^{N} \operatorname{dim} \mathrm{H}_{\mathbb{Q}_{n}}^{j}\left(\mathrm{G}^{\times n}(S) ; \mathbb{Z}_{2}\right) & =P_{N, n}(0)+P_{N, n}^{\prime}(0)+\frac{1}{2} P_{N, n}^{\prime \prime}(0)+\cdots+\frac{1}{N !} P_{N, n}^{(N)}(0) \\
& =N+1 .
\end{aligned}
$$

The iteration theory developed in the current paper is thus needed to conclude that, by varying $n$ in the set of powers of any given prime number, the $n$-periodic billiard trajectories that are found by Morse theory are not all iterations of a finite numbers of lower periodic ones.

Beside the considerations on the Morse coindex, by choosing $p=2$ in Theorem 1.1 we obtain that every convex billiard table admits infinitely many (geometrically distinct) periodic billiard trajectories whose numbers of bounce points are powers of 2. To the best of the author's knowledge, this assertion does not follows from any known multiplicity result in the literature.

Organization of the paper. In Section 2 we recall the basic definitions concerning billiards and the variational principle for periodic billiard trajectories. In Section 3 we introduce the iteration theory for periodic billiard trajectories, discussing in the first subsection the behavior of the Morse indices under iteration, and in subsequent ones the behavior of the local homology groups of periodic billiard trajectories under iteration. Section 4 is devoted to the proof of Theorem 1.1.

\section{Preliminaries}

Throughout this paper, $S$ will be a smooth hypersurface in $\mathbb{R}^{N+1}$, where $N \in \mathbb{N}=$ $\{1,2,3, \ldots\}$, enclosing a compact and strictly convex domain $U_{S}$. Hereafter, strict convexity must be intended in the differentiable sense: the second fundamental form of $S$ is everywhere positive definite. In particular, $S$ is diffeomorphic to the unit $N$-sphere $S^{N} \subset \mathbb{R}^{N+1}$. We are interested in the billiard dynamics in $U_{S}$. A curve $\gamma$ inside $U_{S}$ is a billiard trajectory if it is a piecewise straight curve with constant speed $|\dot{\gamma}|$, except at the instants $t$ in which it hits the hypersurface $S$, where it bounces according to the usual law of reflection: the component of the velocity that is normal to the boundary instantaneously changes sign, whereas the tangential component is preserved. A billiard trajectory $\gamma$ is periodic with period $T>0$ if it is a curve of the form $\gamma: \mathbb{R} / T \mathbb{Z} \rightarrow U_{S}$.

Billiard periodic orbits are characterized by a well-known variational principle which we are going to recall. For each $n \in \mathbb{N}$, denote by $S^{\times n}$ the $n$-fold product $S \times \cdots \times S$. We consider the open subset $\mathrm{G}^{\times n}(S) \subset S^{\times n}$ given by

$$
\mathrm{G}^{\times n}(S)=\left\{\boldsymbol{q}=\left(q_{0}, \ldots, q_{n-1}\right) \in S^{\times n} \mid q_{j} \neq q_{j+1} \quad \text { for all } j \in \mathbb{Z}_{n}\right\},
$$




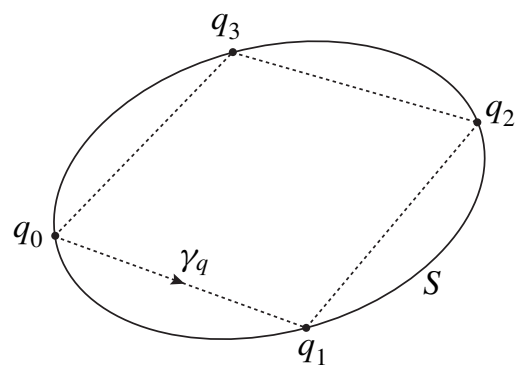

Figure 1. Closed curve $\gamma_{\boldsymbol{q}}$ and corresponding sequence of bounce points $\boldsymbol{q}=\left(q_{0}, \ldots, q_{3}\right)$.

which we will refer to as the cyclic configuration space (or simply the configuration space). The dihedral group $\mathbb{D}_{n}$, seen as a group of permutations of $\mathbb{Z}_{n}$, acts on $\mathrm{G}^{\times n}(S)$ by

$$
\sigma \cdot\left(q_{0}, \ldots, q_{n-1}\right)=\left(q_{\sigma(0)}, \ldots, q_{\sigma(n-1)}\right) \quad \text { for all } \boldsymbol{q} \in \mathrm{G}^{\times n}(S) \text { and } \sigma \in \mathbb{D}_{n} .
$$

For each $\boldsymbol{q} \in \mathrm{G}^{\times n}(S)$, we denote by $\gamma_{\boldsymbol{q}}$ the unique curve in $U_{S}$ with prescribed speed (say, parametrized by arc-length) that is piecewise straight and bounces periodically in the points $q_{0}, \ldots, q_{n-1}$ (see Figure 1). Notice that each point in the $\mathbb{D}_{n}$-orbit of $\boldsymbol{q}$ is associated to the same geometric curve $\gamma_{\boldsymbol{q}}$.

We denote by $L^{\times n}: S^{\times n} \rightarrow \mathbb{R}$ the length functional defined by

$$
L^{\times n}(\boldsymbol{q})=\sum_{j \in \mathbb{Z}_{n}}\left|q_{j+1}-q_{j}\right| \text { for all } \boldsymbol{q}=\left(q_{0}, \ldots, q_{n-1}\right) \in S^{\times n},
$$

namely, $L^{\times n}(\boldsymbol{q})$ is the length of the closed curve $\gamma_{\boldsymbol{q}}$. This functional is continuous, and it is smooth on the configuration space $\mathrm{G}^{\times n}(S)$, whereas is not even differentiable in the complement of $\mathrm{G}^{\times n}(S)$ (since norms are not differentiable at the origin). A straightforward computation shows that

$\mathrm{d} L^{\times n}(\boldsymbol{q}) \boldsymbol{v}=\sum_{j \in \mathbb{Z}_{n}}\langle\underbrace{\frac{q_{j}-q_{j-1}}{\left|q_{j}-q_{j-1}\right|}-\frac{q_{j+1}-q_{j}}{\left|q_{j+1}-q_{j}\right|}}_{q_{j}^{\prime}}, v_{j}\rangle$ for all $\boldsymbol{q} \in \mathrm{G}^{\times n}(S), \boldsymbol{v} \in \mathrm{T}_{\boldsymbol{q}} S^{\times n}$.

A point $\boldsymbol{q} \in \mathrm{G}^{\times n}(S)$ is then a critical point of the length functional $L^{\times n}$ if and only if, for each $j \in \mathbb{Z}_{n}$, the associated point $q_{j}^{\prime} \in \mathbb{R}^{N+1}$ is orthogonal to the tangent space $\mathrm{T}_{q_{j}} S$. This amounts to requiring that the curve $\gamma_{q}$ satisfies the reflection law at the bounce points, and therefore $\boldsymbol{q}$ is a critical point of $L^{\times n}$ if and only $\gamma_{\boldsymbol{q}}$ is a periodic billiard trajectory.

Having this variational principle, one is tempted to study the multiplicity of periodic billiard trajectories with a prescribed number $n$ of bounce points by means 
of critical point theory, more specifically by means of Morse theory or LyusternikSchnirelmann theory for the length functional $L^{\times n}: \mathrm{G}^{\times n}(S) \rightarrow \mathbb{R}$. However, one immediately faces the problem of the lack of compactness of the configuration space $\mathrm{G}^{\times n}(S)$. A possible solution was suggested by Farber and Tabachnikov [2002b], who extended earlier work for the two-dimensional case [Croft and Swinnerton-Dyer 1963; Babenko 1990; Kozlov and Treshchëv 1991]. The idea is to restrict $L^{\times n}$ to the compact subspace $\mathrm{G}_{\epsilon}^{\times n}(S) \subset \mathrm{G}^{\times n}(S)$, where $\epsilon>0$ and

$$
\mathrm{G}_{\epsilon}^{\times n}(S)=\left\{\boldsymbol{q} \in \mathrm{G}^{\times n}(S)\left|\prod_{j \in \mathbb{Z}_{n}}\right| q_{j}-q_{j-1} \mid \geq \epsilon^{n}\right\},
$$

by virtue of the following statement.

Proposition 2.1 [Farber and Tabachnikov 2002b, Proposition 4.1]. For each sufficiently small $\epsilon>0$ the following claims hold:

(i) $\mathrm{G}_{\epsilon}^{\times n}(S)$ is a smooth manifold with boundary.

(ii) The inclusion $\mathrm{G}_{\epsilon}^{\times n}(S) \subset \mathrm{G}^{\times n}(S)$ is a homotopy equivalence.

(iii) All the critical points of $L^{\times n}: \mathrm{G}^{\times n}(S) \rightarrow \mathbb{R}$ are contained in $\mathrm{G}_{\epsilon}^{\times n}(S)$.

(iv) At every point of $\partial \mathrm{G}_{\epsilon}^{\times n}(S)$, the gradient of $L^{\times n}$ points inward.

This proposition guarantees that, for any $\epsilon>0$ sufficiently small, the functional $L^{\times n}: \mathrm{G}_{\epsilon}^{\times n}(S) \rightarrow \mathbb{R}$ satisfies the so-called "general boundary conditions" for Morse theory, see [Chang 1993, Section 6.1]. Moreover, if we perform Morse theory using the gradient flow of $L^{\times n}$ (instead of its antigradient flow, as it would be more common), point (iv) of the proposition implies that the boundary of $\mathrm{G}_{\epsilon}^{\times n}(S)$ does not enter into play while applying the principles of Morse theory.

The homology and cohomology of cyclic configuration spaces have been studied by many authors; see [Arnold 1969; Cohen 1988; Cohen 1995; Farber and Tabachnikov 2002b], for example. In particular, in [Farber and Tabachnikov 2002b] the cohomology and the $\mathbb{D}_{n}$-equivariant cohomology rings of $\mathrm{G}^{\times n}(S)$ with $\mathbb{Z}_{2}$ coefficients have been completely determined. For our purposes, we only need to recall that the Poincaré polynomial of $\mathrm{H}_{*}\left(\mathrm{G}^{\times n}(S) ; \mathbb{Z}_{2}\right)$, defined by

$$
B_{N, n}(t)=\sum_{j=0}^{n N} \operatorname{dim} \mathrm{H}_{j}\left(\mathrm{G}^{\times n}(S) ; \mathbb{Z}_{2}\right) t^{j}
$$

is equal to

$$
B_{N, n}(t)=\frac{\left(t^{N}+1\right)\left(t^{(n-1)(N-1)}-1\right)}{t^{N-1}-1}=\left(t^{N}+1\right)\left(\sum_{j=0}^{n-2} t^{(N-1) j}\right) ;
$$

see [Farber and Tabachnikov 2002b, Theorem 4 and Remarks 3.2 and 3.3]. 


\section{Iteration theory for periodic billiard trajectories}

Morse indices of iterated periodic billiard trajectories. For each $n, m \in \mathbb{N}$, consider the embedding $\psi^{\times m}: S^{\times n} \hookrightarrow S^{\times n m}$ given by

$$
\psi^{\times m}(\boldsymbol{q})=\boldsymbol{q}^{\times m}:=(\underbrace{\boldsymbol{q}, \ldots, \boldsymbol{q}}_{\times m}) \text { for all } \boldsymbol{q} \in S^{\times n} .
$$

This map is clearly smooth (being the restriction of the linear "diagonal" embed$\left.\operatorname{ding} \mathbb{R}^{n N} \hookrightarrow \mathbb{R}^{n m N}\right)$, and it restricts as a map $\mathrm{G}^{\times n}(S) \hookrightarrow \mathrm{G}^{\times n m}(S)$ that we will still denote by $\psi^{\times m}$. This latter map has a clear interpretation in terms of piecewise straight closed curves associated to the points of the configuration spaces: if $\gamma_{q}$ and $\gamma_{\boldsymbol{q}^{\times m}}$ are the closed curves associated to $\boldsymbol{q}$ and $\boldsymbol{q}^{\times m}$ respectively, then $\gamma_{\boldsymbol{q}^{\times m}}$ is the $m$-fold iterate of $\gamma_{\boldsymbol{q}}$. For this reason we call $\psi^{\times m}$ the ( $m$-fold) iteration map.

By the characterization of the critical points of the length functional as bounce points of periodic billiard trajectories, it is clear that $\boldsymbol{q} \in \mathrm{G}^{\times n}(S)$ is a critical point of $L^{\times n}$ if an only if, for some (and thus for all) $m \in \mathbb{N}$, its iteration $\boldsymbol{q}^{\times m}$ is a critical point of $L^{\times n m}$. We denote by $\operatorname{ind}(\boldsymbol{q}), \operatorname{coind}(\boldsymbol{q})$ and $\operatorname{nul}(\boldsymbol{q})$ the Morse index, the Morse coindex and the nullity of the length functional $L^{\times n}$ at $\boldsymbol{q}$. We recall that these are nonnegative integers defined respectively as the dimension of the negative eigenspace, of the positive eigenspace and of the kernel of the Hessian of $L^{\times n}$ at $\boldsymbol{q}$. In this section we investigate the properties of the sequences $\left\{\operatorname{ind}\left(\boldsymbol{q}^{\times m}\right) \mid m \in \mathbb{N}\right.$ \}, $\left\{\operatorname{coind}\left(\boldsymbol{q}^{\times m}\right) \mid m \in \mathbb{N}\right\}$ and $\left\{\operatorname{nul}\left(\boldsymbol{q}^{\times m}\right) \mid m \in \mathbb{N}\right\}$. This of course is reminiscent of the iteration theory for the Morse indices of closed geodesics, which was essentially pioneered by Bott in his celebrated paper [1956], and further extended by many authors also to more general Maslov-type indices; see [Long 2002, Part IV] and the bibliography therein for a detailed account. The results that we are going to present can be considered as discrete-time versions of those contained in [Bott 1956, Section 1]. In view of the application to the multiplicity of periodic billiard trajectories in Section 4 we draw, as a consequence of this iteration theory, the following iteration inequalities analogous to the one established by Liu and Long [1998; 2000] (see also [Long 2002, page 213]) for a Maslov-type index.

Proposition 3.1 (iteration inequalities). Let $\boldsymbol{q}$ be a critical point of $L^{\times n}$.

(i) The nullity of the iterations of $\boldsymbol{q}$ is uniformly bounded by $2 N$, i.e.,

$$
\operatorname{nul}\left(\boldsymbol{q}^{\times m}\right) \leq 2 N
$$

(ii) There is a nonnegative real number $\overline{\operatorname{ind}}(\boldsymbol{q})$, the mean Morse index of $L^{\times n}$ at $\boldsymbol{q}$, defined by

$$
\overline{\operatorname{ind}}(\boldsymbol{q})=\lim _{m \rightarrow \infty} \frac{1}{m} \operatorname{ind}\left(\boldsymbol{q}^{\times m}\right),
$$


such that

$$
m \overline{\operatorname{ind}}(\boldsymbol{q})-2 N \leq \operatorname{ind}\left(\boldsymbol{q}^{\times m}\right) \leq m \overline{\operatorname{ind}}(\boldsymbol{q})+2 N-\operatorname{nul}\left(\boldsymbol{q}^{\times m}\right) .
$$

Analogously, there is a nonnegative real number $\overline{\operatorname{coind}}(\boldsymbol{q})$, the mean Morse coindex of $L^{\times n}$ at $\boldsymbol{q}$, defined by

$$
\overline{\operatorname{coind}}(\boldsymbol{q})=\lim _{m \rightarrow \infty} \frac{1}{m} \operatorname{coind}\left(\boldsymbol{q}^{\times m}\right),
$$

such that

$$
m \overline{\operatorname{coind}}(\boldsymbol{q})-2 N \leq \operatorname{coind}\left(\boldsymbol{q}^{\times m}\right) \leq m \overline{\operatorname{coind}}(\boldsymbol{q})+2 N-\operatorname{nul}\left(\boldsymbol{q}^{\times m}\right) .
$$

The proof of this proposition will be carried out at the end of this subsection. The reader might skip the following paragraphs and go directly to the middle of page 193 on a first reading.

To begin with, let us write down an expression for the Hessian of $L^{\times n}$ at the critical point $\boldsymbol{q}$. For each $\boldsymbol{v}, \boldsymbol{w} \in \mathrm{T}_{\boldsymbol{q}} S^{\times n}$ we have

$$
\text { hess } \begin{aligned}
L^{\times n}(\boldsymbol{q})[\boldsymbol{v}, \boldsymbol{w}]=\sum_{j \in \mathbb{Z}_{n}} & \frac{1}{\left|q_{j+1}-q_{j}\right|}\left(\left\langle v_{j+1}-v_{j}, w_{j+1}-w_{j}\right\rangle\right. \\
& \left.-\left\langle\frac{q_{j+1}-q_{j}}{\left|q_{j+1}-q_{j}\right|}, v_{j+1}-v_{j}\right\rangle\left\langle\frac{q_{j+1}-q_{j}}{\left|q_{j+1}-q_{j}\right|}, w_{j+1}-w_{j}\right\rangle\right) .
\end{aligned}
$$

Denote by $H=H_{q}$ the self-adjoint endomorphism of $\mathrm{T}_{\boldsymbol{q}} S^{\times n}$ associated to the Hessian of $L^{\times n}$ at $\boldsymbol{q}$, i.e., hess $L^{\times n}(\boldsymbol{q})[\boldsymbol{v}, \boldsymbol{w}]=\langle\boldsymbol{H} \boldsymbol{v}, \boldsymbol{w}\rangle$. If we write

$$
H \boldsymbol{v}=\left((H \boldsymbol{v})_{1}, \ldots,(H \boldsymbol{v})_{n}\right),
$$

then for each $j \in \mathbb{Z}_{n}$ we have

$$
(H \boldsymbol{v})_{j}=-\frac{1}{\left|q_{j+1}-q_{j}\right|} \pi_{j} \circ \tilde{\pi}_{j}\left(v_{j+1}-v_{j}\right)+\frac{1}{\left|q_{j}-q_{j-1}\right|} \pi_{j} \circ \tilde{\pi}_{j-1}\left(v_{j}-v_{j-1}\right),
$$

where $\pi_{j}: \mathbb{R}^{N+1} \rightarrow \mathrm{T}_{q_{j}} S$ and $\tilde{\pi}_{j}: \mathbb{R}^{N+1} \rightarrow\left\langle q_{j+1}-q_{j}\right\rangle^{\perp}$ are orthogonal projectors. This expression shows that $H$ is a second order difference operator. Now, for each $m \in \mathbb{N}$ and $z \in S^{1} \subset \mathbb{C}$, we consider the vector space of sequences $\mathscr{V}_{m, z}$ given by

$$
\mathscr{V}_{m, z}=\left\{\boldsymbol{v}=\left\{v_{j} \mid j \in \mathbb{Z}\right\} \mid v_{j} \in \mathrm{T}_{q_{j}} S \otimes \mathbb{C}, v_{j+n m}=z v_{j} \text { for all } j \in \mathbb{Z}\right\},
$$

and, for each $\lambda \in \mathbb{R}$, the eigenvalue problem

$$
H \boldsymbol{v}=\lambda \boldsymbol{v}, \quad \boldsymbol{v} \in \mathscr{V}_{m, z} .
$$

We denote by $\operatorname{ind}_{z, \lambda}\left(\boldsymbol{q}^{\times m}\right)$ the number of complex-linearly independent solutions of this eigenvalue problem. Notice that, since $H$ is a real operator with real eigenvalues, the sequence $\boldsymbol{v} \in \mathscr{V}_{m, z}$ is a solution of $H \boldsymbol{v}=\lambda \boldsymbol{v}$ if and only of the complex 
conjugate sequence $\overline{\boldsymbol{v}} \in \mathscr{V}_{m, \bar{z}}$ is a solution of $H \overline{\boldsymbol{v}}=\lambda \overline{\boldsymbol{v}}$, and therefore

$$
\operatorname{ind}_{z, \lambda}\left(\boldsymbol{q}^{\times m}\right)=\operatorname{ind}_{\bar{z}, \lambda}\left(\boldsymbol{q}^{\times m}\right) .
$$

Now set

$$
\begin{aligned}
\operatorname{ind}_{z}\left(\boldsymbol{q}^{\times m}\right) & :=\sum_{\lambda<0} \operatorname{ind}_{z, \lambda}\left(\boldsymbol{q}^{\times m}\right), \\
\operatorname{coind}_{z}\left(\boldsymbol{q}^{\times m}\right) & :=\sum_{\lambda>0} \operatorname{ind}_{z, \lambda}\left(\boldsymbol{q}^{\times m}\right), \\
\operatorname{nul}_{z}\left(\boldsymbol{q}^{\times m}\right) & :=\operatorname{ind}_{z, 0}\left(\boldsymbol{q}^{\times m}\right)
\end{aligned}
$$

These sums are finite, for $\operatorname{ind}_{z, \lambda}\left(\boldsymbol{q}^{\times m}\right)$ is different from zero only if $\lambda$ belongs to the (finite) spectrum of $H$. These integer indices that we have just defined generalize the Morse index, the Morse coindex and the nullity for, as it readily follows from their definition, we have

$$
\begin{aligned}
\operatorname{ind}\left(\boldsymbol{q}^{\times m}\right) & =\operatorname{ind}_{1}\left(\boldsymbol{q}^{\times m}\right), \\
\operatorname{coind}\left(\boldsymbol{q}^{\times m}\right) & =\operatorname{coind}_{1}\left(\boldsymbol{q}^{\times m}\right), \\
\operatorname{nul}\left(\boldsymbol{q}^{\times m}\right) & =\operatorname{nul}_{1}\left(\boldsymbol{q}^{\times m}\right) .
\end{aligned}
$$

The reason for considering the eigenvalue problem for the operator $H$ in the complexified setting is that there is a nice way to compute the index $\operatorname{ind}_{z, \lambda}\left(\boldsymbol{q}^{\times m}\right)$ from the indices $\operatorname{ind}_{w, \lambda}(\boldsymbol{q})$, for every $w \in S^{1}$, of the non-iterated critical point. The recipe is given by the following statement which is analogous to [Bott 1956, Theorem I]. Its proof is a simple application of the Fourier expansion of "periodic" sequences, and we include it here for the reader's convenience.

Proposition 3.2 [Bott 1956, Theorem I]. For each $z \in S^{1}, \lambda \in \mathbb{R}$ and $m \in \mathbb{N}$, the index $\operatorname{ind}_{z, \lambda}\left(\boldsymbol{q}^{\times m}\right)$ satisfies

$$
\operatorname{ind}_{z, \lambda}\left(\boldsymbol{q}^{\times m}\right)=\sum_{w \in \sqrt[m]{z}} \operatorname{ind}_{w, \lambda}(\boldsymbol{q}) .
$$

Proof. The operator $H$ maps each vector space $\mathscr{V}_{m, z}$ to itself. In fact, consider the shift operator $S: \mathscr{V}_{m, z} \rightarrow \mathscr{V}_{m, z}$ given by

$$
(S \boldsymbol{v})_{j}=v_{j+n} \quad \text { for all } \boldsymbol{v}=\left\{v_{j} \mid j \in \mathbb{Z}\right\} \in \mathscr{V}_{m, z} .
$$

By the definition of $H$ (in particular, notice that the index $j$ in (3-1) is defined modulo $n$ ) we have that $S H=H S$. Moreover, since $H$ is a real operator, we have that

$$
z H \boldsymbol{v}=H z \boldsymbol{v}=H S^{m} \boldsymbol{v}=S^{m} H \boldsymbol{v} \quad \text { for all } \boldsymbol{v} \in \mathscr{V}_{m, z},
$$

which proves the claim. 
Now, every $\boldsymbol{v}=\left\{v_{j} \mid j \in \mathbb{Z}\right\} \in \mathscr{V}_{m, z}$ admits a unique Fourier expansion

$$
\boldsymbol{v}=\sum_{w \in \sqrt[m]{z}} \boldsymbol{v}_{w}
$$

where, for each $w \in \sqrt[m]{z}$, the sequence $\boldsymbol{v}_{w}=\left\{v_{w, j} \mid j \in \mathbb{Z}\right\}$ belongs to the vector space $\mathscr{V}_{1, w}$. One can explicitly compute $\boldsymbol{v}_{w}$ as

$$
v_{w, j}=\frac{1}{m} \sum_{h=0}^{m-1} w^{1-h} v_{j+h} \quad \text { for all } j \in \mathbb{Z} .
$$

By the first part of the proof we have that $H \boldsymbol{v} \in \mathscr{V}_{m, z}$ and $H \boldsymbol{v}_{w} \in \mathscr{V}_{1, w}$. Hence, the unique Fourier expansion of $H \boldsymbol{v} \in \mathscr{V}_{m, z}$ is given by

$$
H \boldsymbol{v}=\sum_{w \in \sqrt[m]{z}} H \boldsymbol{v}_{w}
$$

From this, we conclude that $\boldsymbol{v}$ satisfies the eigenvalue problem $H \boldsymbol{v}=\lambda \boldsymbol{v}$ if and only if all the $\boldsymbol{v}_{w}$ 's satisfy the same eigenvalue problem $H \boldsymbol{v}_{w}=\lambda \boldsymbol{v}_{w}$.

This proposition tells us that it is enough to study the indices of $\boldsymbol{q}^{\times m}$ with a fixed $m \in \mathbb{N}$, say $m=1$. To start with, let us investigate the properties of $\operatorname{nul}_{z}(\boldsymbol{q})$. We call $z \in S^{1}$ a Poincaré point of $\boldsymbol{q}$ when $\operatorname{nul}_{z}(\boldsymbol{q}) \neq 0$.

Proposition 3.3. There are only finitely many Poincaré points $z_{1}, \ldots, z_{r} \in S^{1}$ and we have

$$
\sum_{\alpha=1}^{r} \operatorname{nul}_{z_{\alpha}}(\boldsymbol{q}) \leq 2 N .
$$

Proof. Let $\boldsymbol{v}=\left\{v_{j}\right\}$ be a sequence such that, for each $j \in \mathbb{Z}$, the element $v_{j}$ belongs to the tangent space $\mathrm{T}_{q_{j}} S$. By (3-1), $v$ satisfies $H \boldsymbol{v}=0$ if and only if

$$
\frac{1}{\left|q_{j+1}-q_{j}\right|} \pi_{j} \circ \tilde{\pi}_{j}\left(v_{j+1}-v_{j}\right)=\frac{1}{\left|q_{j}-q_{j-1}\right|} \pi_{j} \circ \tilde{\pi}_{j-1}\left(v_{j}-v_{j-1}\right)
$$

for all $z \in \mathbb{Z}$,

where $\pi_{j}: \mathbb{R}^{N+1} \rightarrow \mathrm{T}_{q_{j}} S$ and $\tilde{\pi}_{j}: \mathbb{R}^{N+1} \rightarrow\left\langle q_{j+1}-q_{j}\right\rangle^{\perp}$ are orthogonal projectors as above. For our convenience, let us rewrite (3-3) as

$$
\pi_{j} \circ \tilde{\pi}_{j}\left(v_{j+1}\right)=\pi_{j} \circ \tilde{\pi}_{j}\left(v_{j}\right)+\frac{\left|q_{j+1}-q_{j}\right|}{\left|q_{j}-q_{j-1}\right|} \pi_{j} \circ \tilde{\pi}_{j-1}\left(v_{j}-v_{j-1}\right) .
$$

Now, since the vector $q_{j+1}-q_{j} \in \mathbb{R}^{N+1}$ is transverse to $\mathrm{T}_{q_{j+1}} S$ (as well as to $\mathrm{T}_{q_{j}} S$ ), the composition $\pi_{j} \circ \tilde{\pi}_{j}$ restricts to an isomorphism

$$
\mathrm{T}_{q_{j+1}} S \stackrel{\cong}{\longrightarrow} \mathrm{T}_{q_{j}} S .
$$


This shows that we can rewrite (3-4) as

$$
v_{j+1}=A_{j} v_{j}+B_{j} v_{j-1},
$$

where $A_{j}: \mathrm{T}_{q_{j}} S \rightarrow \mathrm{T}_{q_{j+1}} S$ and $B_{j}: \mathrm{T}_{q_{j-1}} S \rightarrow \mathrm{T}_{q_{j+1}} S$ are linear maps. Analogously, we can rewrite (3-4) as

$$
v_{j-1}=C_{j} v_{j+1}+D_{j} v_{j}
$$

where $C_{j}: \mathrm{T}_{q_{j+1}} S \rightarrow \mathrm{T}_{q_{j-1}} S$ and $D_{j}: \mathrm{T}_{q_{j}} S \rightarrow \mathrm{T}_{q_{j-1}} S$ are linear maps. Equations (3-5) and (3-6) show that every solution $\boldsymbol{v}=\left\{v_{j}\right\}$ of $H \boldsymbol{v}=0$ is completely determined by two of its subsequent points, say $\left(v_{0}, v_{1}\right)$, and conversely any choice of $\left(v_{0}, v_{1}\right)$ uniquely determine a solution $\boldsymbol{v}$. Moreover, $\boldsymbol{v}$ depends linearly on $\left(v_{0}, v_{1}\right)$. Denote by $\Phi$ the linear endomorphism of $\mathrm{T}_{q_{0}} S \oplus \mathrm{T}_{q_{1}} S$ given by $\Phi\left(v_{0}, v_{1}\right)=$ $\left(v_{n}, v_{n+1}\right)$, and let us extend it as a complex linear endomorphism of $\left(\mathrm{T}_{q_{0}} S \oplus\right.$ $\left.\mathrm{T}_{q_{1}} S\right) \otimes \mathbb{C}$.

Now, let us take $z \in S^{1}$. By its definition, the integer $\operatorname{nul}_{z}(\boldsymbol{q})$ is equal to the complex dimension of the kernel of $(\Phi-z \mathrm{Id})$. This establishes the proposition.

Remark. If $z_{1}, \ldots, z_{r}$ are the Poincaré points of $\boldsymbol{q}$, their $m$-th powers $z_{1}^{m}, \ldots, z_{r}^{m}$ are the Poincaré points of $\boldsymbol{q}^{\times m}$, for each $m \in \mathbb{N}$.

The next statement summarizes the properties of $\operatorname{ind}_{z}(\boldsymbol{q})$ and $\operatorname{coind}_{z}(\boldsymbol{q})$.

Proposition 3.4. The functions $z \mapsto \operatorname{ind}_{z}(\boldsymbol{q})$ and $z \mapsto \operatorname{coind}_{z}(\boldsymbol{q})$ are locally constant on $S^{1} \backslash\left\{z_{1}, \ldots, z_{r}\right\}$, where $z_{1}, \ldots, z_{r}$ are the Poincaré points, and lower semicontinuous on $S^{1}$. Moreover, the jump of these functions at any Poincaré point $z_{\alpha}$ is bounded in absolute value by $\operatorname{nul}_{z_{\alpha}}(\boldsymbol{q})$, i.e.,

$$
\begin{gathered}
\operatorname{ind}_{z_{\alpha}}(\boldsymbol{q}) \leq \lim _{z \rightarrow z_{\alpha}^{ \pm}} \operatorname{ind}_{z}(\boldsymbol{q}) \leq \operatorname{ind}_{z_{\alpha}}(\boldsymbol{q})+\operatorname{nul}_{z_{\alpha}}(\boldsymbol{q}), \\
\operatorname{coind}_{z_{\alpha}}(\boldsymbol{q}) \leq \lim _{z \rightarrow z_{\alpha}^{ \pm}} \operatorname{coind}_{z}(\boldsymbol{q}) \leq \operatorname{coind}_{z_{\alpha}}(\boldsymbol{q})+\operatorname{nul}_{z_{\alpha}}(\boldsymbol{q}) .
\end{gathered}
$$

Proof. For each $z \in S^{1}$, let us denote by $\sigma_{z} \subset \mathbb{R}$ the spectrum of the operator $H: \mathscr{V}_{1, z} \rightarrow \mathscr{V}_{1, z}$. This spectrum satisfies the following continuity property: for each interval $(a, b) \subset \mathbb{R} \cup\{ \pm \infty\}$ such that $a$ and $b$ do not belong to $\sigma_{z}$, there is a neighborhood of $z$ in $S^{1}$ such that, for each $z^{\prime}$ in this neighborhood, $a$ and $b$ do not belong to $\sigma_{z^{\prime}}$ and moreover

$$
\sum_{\lambda \in(a, b)} \operatorname{ind}_{z, \lambda}(\boldsymbol{q})=\sum_{\lambda \in(a, b)} \operatorname{ind}_{z^{\prime}, \lambda}(\boldsymbol{q}) .
$$

Now, assume that $z \in S^{1}$ is not a Poincaré point, so 0 does not belong to $\sigma_{z}$. Then, by the continuity property above, 0 does not belong to $\sigma_{z^{\prime}}$ for each $z^{\prime}$ in some 
neighborhood of $z$, and moreover

$$
\operatorname{ind}_{z}(\boldsymbol{q})=\sum_{\lambda<0} \operatorname{ind}_{z, \lambda}(\boldsymbol{q})=\sum_{\lambda<0} \operatorname{ind}_{z^{\prime}, \lambda}(\boldsymbol{q})=\operatorname{ind}_{z^{\prime}}(\boldsymbol{q}) .
$$

Finally, assume that $z \in S^{1}$ is a Poincaré point, and let us fix a sufficiently small $\epsilon>0$ so that $[-\epsilon, \epsilon] \cap \sigma_{z}=\{0\}$. Then, by the above continuity property, $-\epsilon$ and $\epsilon$ do not belong to $\sigma_{z^{\prime}}$ for each $z^{\prime}$ in some neighborhood of $z$, and we have

$$
\begin{aligned}
\operatorname{ind}_{z^{\prime}}(\boldsymbol{q}) & =\sum_{\lambda<-\epsilon} \operatorname{ind}_{z^{\prime}, \lambda}(\boldsymbol{q})+\sum_{\lambda \in(-\epsilon, 0)} \operatorname{ind}_{z^{\prime}, \lambda}(\boldsymbol{q}) \\
& =\sum_{\lambda<-\epsilon} \operatorname{ind}_{z, \lambda}(\boldsymbol{q})+\sum_{\lambda \in(-\epsilon, 0)} \operatorname{ind}_{z^{\prime}, \lambda}(\boldsymbol{q}) \\
& =\operatorname{ind}_{z}(\boldsymbol{q})+\sum_{\lambda \in(-\epsilon, 0)} \operatorname{ind}_{z^{\prime}, \lambda}(\boldsymbol{q}) .
\end{aligned}
$$

This proves that $\operatorname{ind}_{z}(\boldsymbol{q}) \leq \operatorname{ind}_{z^{\prime}}(\boldsymbol{q})$. Moreover

$$
\begin{aligned}
\operatorname{ind}_{z^{\prime}}(\boldsymbol{q}) & =\operatorname{ind}_{z}(\boldsymbol{q})+\sum_{\lambda \in(-\epsilon, 0)} \operatorname{ind}_{z^{\prime}, \lambda}(\boldsymbol{q}) \\
& \leq \operatorname{ind}_{z}(\boldsymbol{q})+\sum_{\lambda \in(-\epsilon, \epsilon)} \operatorname{ind}_{z^{\prime}, \lambda}(\boldsymbol{q}) \\
& =\operatorname{ind}_{z}(\boldsymbol{q})+\sum_{\lambda \in(-\epsilon, \epsilon)} \operatorname{ind}_{z, \lambda}(\boldsymbol{q}) \\
& =\operatorname{ind}_{z}(\boldsymbol{q})+\operatorname{nul}_{z}(\boldsymbol{q}) .
\end{aligned}
$$

The statement regarding $\operatorname{coind}_{z}(\boldsymbol{q})$ is established in the same way.

Proof of Proposition 3.1. Point (i) follows from Propositions 3.2 and 3.3. As for point (ii), let us fix $m \in \mathbb{N}$ and denote by $z_{1}, \ldots, z_{r} \in S^{1}$ the Poincaré points of $\boldsymbol{q}$, so that $z_{1}^{m}, \ldots, z_{r}^{m}$ are the Poincaré points of $\boldsymbol{q}^{\times m}$. By Proposition 3.4, for each $w, z \in S^{1}$ we have

$$
\operatorname{ind}_{w}\left(\boldsymbol{q}^{\times m}\right)+\operatorname{nul}_{w}\left(\boldsymbol{q}^{\times m}\right) \leq \operatorname{ind}_{z}\left(\boldsymbol{q}^{\times m}\right)+\sum_{\alpha=1}^{r} \operatorname{nul}_{z_{\alpha}^{m}}\left(\boldsymbol{q}^{\times m}\right)
$$

By Propositions 3.2 and 3.3 we have

$$
\sum_{\alpha=1}^{r} \operatorname{nul}_{z_{\alpha}^{m}}\left(\boldsymbol{q}^{\times m}\right)=\sum_{\alpha=1}^{r} \operatorname{nul}_{z_{\alpha}}(\boldsymbol{q}) \leq 2 N,
$$

and, together with (3-7), we obtain

$$
\operatorname{ind}_{w}\left(\boldsymbol{q}^{\times m}\right)+\operatorname{nul}_{w}\left(\boldsymbol{q}^{\times m}\right) \leq \operatorname{ind}_{z}\left(\boldsymbol{q}^{\times m}\right)+2 N .
$$


Now, by Propositions 3.2 and 3.4, we have

$$
\overline{\operatorname{ind}}(\boldsymbol{q})=\lim _{m \rightarrow \infty} \frac{\operatorname{ind}\left(\boldsymbol{q}^{\times m}\right)}{m}=\lim _{m \rightarrow \infty} \frac{1}{m} \sum_{w^{m}=1} \operatorname{ind}_{w}(\boldsymbol{q})=\frac{1}{2 \pi} \int_{0}^{2 \pi} \operatorname{ind}_{e^{i \theta}}(\boldsymbol{q}) \mathrm{d} \theta .
$$

Notice that

$$
\frac{1}{2 \pi} \int_{0}^{2 \pi} \operatorname{ind}_{e^{i \theta}}\left(\boldsymbol{q}^{\times m}\right) \mathrm{d} \theta=\overline{\operatorname{ind}}\left(\boldsymbol{q}^{\times m}\right)=m \overline{\operatorname{ind}}(\boldsymbol{q}),
$$

and moreover, since $\operatorname{nul}_{z}\left(\boldsymbol{q}^{\times m}\right)=0$ for every $z \in S^{1} \backslash\left\{z_{1}^{m}, \ldots, z_{r}^{m}\right\}$, we have

$$
\frac{1}{2 \pi} \int_{0}^{2 \pi} \operatorname{nul}_{e^{i \theta}\left(\boldsymbol{q}^{\times m}\right) \mathrm{d} \theta=0 .}
$$

Now, by setting $z=1$ and integrating $w$ on $S^{1}$ in (3-8), we get

$$
m \overline{\operatorname{ind}}(\boldsymbol{q}) \leq \operatorname{ind}\left(\boldsymbol{q}^{\times m}\right)+2 N .
$$

Then, by setting $w=1$ and integrating $z$ on $S^{1}$ in (3-8), we get

$$
\operatorname{ind}\left(\boldsymbol{q}^{\times m}\right)+\operatorname{nul}\left(\boldsymbol{q}^{\times m}\right) \leq m \overline{\operatorname{ind}}(\boldsymbol{q})+2 N .
$$

This proves point (ii) for the Morse index. The proof for the Morse coindex is analogous.

Local homology of iterated periodic billiard trajectories. This section is devoted to prove that, if the Morse indices of an isolated critical point of the length functional are preserved by iteration, then the same is true for its local homology. As for the previous subsection, there is a clear parallel with the theory of closed geodesics: in fact, this result has been established for closed geodesics by Gromoll and Meyer [1969b], and further extended to more general Lagrangian systems by Long [2000], $\mathrm{Lu}$ [2009] and the author [Mazzucchelli 2011]. In the following, we prove the result after recalling the notion of local homology.

From now on, all the homology groups are assumed to have coefficients in $\mathbb{Z}_{2}$. For technical reasons (see the discussion after Proposition 2.1) we will work with minus the length functional, that is, with superlevels of the length functional. The local homology of $-L^{\times n}$ at an isolated critical point $\boldsymbol{q}$ is the homology group $\mathrm{C}_{*}(\boldsymbol{q})$ defined by

$$
\mathrm{C}_{*}(\boldsymbol{q}):=\mathrm{H}_{*}\left(\mathrm{G}^{\times n}(S)_{>c} \cup\{\boldsymbol{q}\}, \mathrm{G}^{\times n}(S)_{>c}\right),
$$

where $c=L^{\times n}(\boldsymbol{q})$ and $\mathrm{G}^{\times n}(S)_{>c}=\left\{\boldsymbol{q}^{\prime} \in \mathrm{G}^{\times n}(S) \mid L^{\times n}\left(\boldsymbol{q}^{\prime}\right)>c\right\}$. Recall that the dihedral group $\mathbb{D}_{n}$ acts by coordinates-permutations on the cyclic configuration space $\mathrm{G}^{\times n}(S)$ and $L^{\times n}$ is invariant under its action, see Section 2. The local homology of $-L^{\times n}$ at the $\mathbb{D}_{n}$-orbit of $\boldsymbol{q}$ is defined by

$$
\mathrm{C}_{*}\left(\mathbb{D}_{n} \cdot\{\boldsymbol{q}\}\right):=\mathrm{H}_{*}\left(\mathrm{G}^{\times n}(S)_{>c} \cup \mathbb{D}_{n} \cdot\{\boldsymbol{q}\}, \mathrm{G}^{\times n}(S)_{>c}\right) .
$$


By excision, it is straightforward to verify that the local homology of $\mathbb{D}_{n} \cdot\{\boldsymbol{q}\}$ is the direct sum of the local homology of each element in the $\mathbb{D}_{n}$-orbit of $\boldsymbol{q}$, and in particular the inclusion induces a homology monomorphism

$$
\mathrm{C}_{*}(\boldsymbol{q}) \hookrightarrow \mathrm{C}_{*}\left(\mathbb{D}_{n} \cdot\{\boldsymbol{q}\}\right) .
$$

We also recall that the local homology $\mathrm{C}_{k}(\boldsymbol{q})$, and therefore $\mathrm{C}_{k}\left(\mathbb{D}_{n} \cdot\{\boldsymbol{q}\}\right)$ as well, is possibly nontrivial only if $\operatorname{coind}(\boldsymbol{q}) \leq k \leq \operatorname{coind}(\boldsymbol{q})+\operatorname{nul}(\boldsymbol{q})$, and that $\boldsymbol{q}$ is a local maximum of $L^{\times n}$ if and only if

$$
\mathrm{C}_{k}(\boldsymbol{q})= \begin{cases}\mathbb{Z}_{2} & \text { if } k=0 \\ 0 & \text { if } k \neq 0\end{cases}
$$

For a proof of these results as well as for more details on the local homology groups, see [Gromoll and Meyer 1969a] or [Chang 1993, Chapter I]. The reader should keep in mind that the Morse coindex of $L^{\times n}$ is the Morse index of $-L^{\times n}$ and vice versa.

The main result of this subsection is the following.

Proposition 3.5. Let $\boldsymbol{q} \in \mathrm{G}^{\times n}(S)$ be an isolated critical point of $L^{\times n}$ with critical value $L^{\times n}(\boldsymbol{q})=c$, and assume that, for some $m \in \mathbb{N}$, we have

$$
\operatorname{coind}(\boldsymbol{q})=\operatorname{coind}\left(\boldsymbol{q}^{\times m}\right), \quad \operatorname{nul}(\boldsymbol{q})=\operatorname{nul}\left(\boldsymbol{q}^{\times m}\right) .
$$

Then, the iteration map $\psi^{\times m}$ induces the homology isomorphism

$$
\psi_{*}^{\times m}: \mathrm{C}_{*}(\boldsymbol{q}) \stackrel{\cong}{\longrightarrow} \mathrm{C}_{*}\left(\boldsymbol{q}^{\times m}\right) .
$$

We will prove this proposition by means of the following abstract principle of Morse theory. Here we only quote the statement for a finite-dimensional setting, as needed for our purposes.

Proposition 3.6 [Mazzucchelli 2011, Theorem 4.1]. Let $U \subseteq \mathbb{R}^{k}$ be an open neighborhood of the origin, $F: U \rightarrow \mathbb{R}$ a smooth functional having the origin as isolated critical point, and $\mathbb{V} \subset \mathbb{R}^{k}$ a vector subspace. Assume that $\nabla F(x) \in \mathbb{V}$ for each $x \in U \cap \mathbb{V}$, and that the Morse index and the nullity of $F$ at the origin are equal to the Morse index and the nullity of the restricted functional $\left.F\right|_{U \cap \vee}$ at the origin. Then the inclusion $U \cap \mathbb{V} \subset U$ induces an isomorphism between the local homology of $\left.F\right|_{U \cap V}$ at the origin and the local homology of $F$ at the origin.

Proof of Proposition 3.5. All we need to do is to reduce our setting in such a way that we satisfy the assumptions of Proposition 3.6. For each $j \in \mathbb{Z}_{n}$ let us consider a chart $\phi_{j}: V_{j} \rightarrow \mathbb{R}^{N}$ for $S$, where $V_{j}$ is an open neighborhood of $q_{j}$. Up to shrink the $V_{j}$ 's, we can assume that $V_{j} \cap V_{j+1}=\varnothing$. We further define smooth functions 
$\ell_{j}: V_{j} \times V_{j+1} \rightarrow \mathbb{R}$ by

$$
\ell_{j}\left(x_{j}, x_{j+1}\right)=\left|\phi_{j}^{-1}\left(x_{j}\right)-\phi_{j+1}^{-1}\left(x_{j+1}\right)\right| \quad \text { for all }\left(x_{j}, x_{j+1}\right) \in V_{j} \times V_{j+1} .
$$

Then, the product $V:=V_{0} \times \cdots \times V_{n-1}$ is an open set of $\mathrm{G}^{\times n}(S)$, and the map

$$
\phi:=\left(\phi_{0}, \ldots, \phi_{n-1}\right): V \rightarrow \mathbb{R}^{n N}
$$

is a chart for $\mathrm{G}^{\times n}(S)$ centered at $\boldsymbol{q}$. In this local coordinates the length functional $L^{\times n}$ can be written as

$\ell^{\times n}(\boldsymbol{x}):=L^{\times n} \circ \boldsymbol{\phi}^{-1}(\boldsymbol{x})=\sum_{j \in \mathbb{Z}_{n}} \ell_{j}\left(x_{j}, x_{j+1}\right) \quad$ for all $\boldsymbol{x}=\left(x_{0}, \ldots, x_{n-1}\right) \in \boldsymbol{\phi}(V)$.

A straightforward computation shows that the gradient of $\ell^{\times n}$ at $x$, with respect to the flat metric of $\mathbb{R}^{n N}$, is given by $\boldsymbol{g}=\left(g_{0}, \ldots, g_{n-1}\right)$, where

$$
g_{j}=\partial_{1} \ell_{j}\left(x_{j}, x_{j+1}\right)+\partial_{2} \ell_{j-1}\left(x_{j-1}, x_{j}\right) \text { for all } j \in \mathbb{Z}_{n} .
$$

Now, consider the map

$$
\boldsymbol{\phi}^{\times m}=(\underbrace{\boldsymbol{\phi}, \ldots, \boldsymbol{\phi}}_{\times m}): V^{\times m} \rightarrow\left(\mathbb{R}^{n N}\right)^{\times m} .
$$

This map is a chart for $\mathrm{G}^{\times n m}(S)$ centered at $\boldsymbol{q}^{\times m}$, and in this local coordinates we denote the length functional $L^{\times n m}$ by $\ell^{\times n m}:=L^{\times n m} \circ\left(\boldsymbol{\phi}^{\times m}\right)^{-1}$. Now, if we put on $\left(\mathbb{R}^{n N}\right)^{\times m}$ the flat metric rescaled by the factor $m^{-1}$, the gradient of $\ell^{\times n m}$ at $\boldsymbol{y}$ is given by $\tilde{\boldsymbol{g}}=\left(\tilde{g}_{0}, \ldots, \tilde{g}_{n m-1}\right)$, where

$$
\tilde{g}_{j}=\partial_{1} \ell_{j \bmod n}\left(y_{j}, y_{j+1}\right)+\partial_{2} \ell_{j-1 \bmod n}\left(y_{j-1}, y_{j}\right) \text { for all } j \in \mathbb{Z}_{n m} .
$$

Denote by $\Psi^{\times m}:=\boldsymbol{\phi}^{\times m} \circ \psi^{\times m} \circ \boldsymbol{\phi}^{-1}$ the iteration map in local coordinates, which turns out to be the restriction of the (linear) diagonal embedding of $\mathbb{R}^{n N}$ into $\left(\mathbb{R}^{n N}\right)^{\times m}$. Notice that $\ell^{\times n m} \circ \Psi^{\times m}=m \ell^{\times n}$. From this, together with (3-9) and (3-10), we infer

$$
\left(\nabla \ell^{\times n m}\right) \circ \Psi^{\times m}=m \nabla \ell^{\times n} .
$$

Therefore, the claim of the proposition follows by applying Proposition 3.6 with $U=\boldsymbol{\phi}^{\times m}\left(V^{\times m}\right), F=-m^{-1} \ell^{\times n m}$ and the inclusion $U \cap \mathbb{V} \subset U$ given by the iteration map $\Psi^{\times m}$.

Homological vanishing by iteration. In this section we show how to recover, in the "discrete" setting of billiards, the homological vanishing under iteration. This is a remarkable phenomenon, discovered by Bangert [1980, Section 3] in the study of closed geodesics and further investigated in [Bangert and Klingenberg 1983; Long 2000; Lu 2009; Mazzucchelli 2011]. 
Let $\boldsymbol{q}$ be a critical point of the length functional $L^{\times n}$ with critical value $c=$ $L^{\times n}(\boldsymbol{q})$. For each $\epsilon>0$ sufficiently small, the subset $\mathrm{G}_{\epsilon}^{\times n}(S) \subset \mathrm{G}^{\times n}(S)$ defined in (2-1) contains $\boldsymbol{q}$, and therefore for each $m \in \mathbb{N}$ the subset $\mathrm{G}_{\epsilon}^{\times n m}(S) \subset \mathrm{G}^{\times n m}(S)$ contains $\boldsymbol{q}^{\times m}$. Now notice that, by excision, the local homology of $L^{\times n}$ at $\boldsymbol{q}$ can be equivalently defined as

$$
\mathrm{C}_{*}(\boldsymbol{q})=\mathrm{H}_{*}\left(\mathrm{G}_{\epsilon}^{\times n}(S)_{>c} \cup\{\boldsymbol{q}\}, \mathrm{G}_{\epsilon}^{\times n}(S)_{>c}\right) .
$$

Then, let us fix an arbitrary $b<c$ and consider the iteration map restricted as a map of pairs of the form

$$
\psi^{\times m}:\left(\mathrm{G}_{\epsilon}^{\times n}(S)_{>c} \cup\{\boldsymbol{q}\}, \mathrm{G}_{\epsilon}^{\times n}(S)_{>c}\right) \hookrightarrow\left(\mathrm{G}_{\epsilon}^{\times n m}(S)_{>m b}, \mathrm{G}_{\epsilon}^{\times n m}(S)_{>m c}\right) .
$$

In homology, this map induces the homomorphism

$$
\psi_{*}^{\times m}: \mathrm{C}_{*}(\boldsymbol{q}) \rightarrow \mathrm{H}_{*}\left(\mathrm{G}_{\epsilon}^{\times n m}(S)_{>m b}, \mathrm{G}_{\epsilon}^{\times n m}(S)_{>m c}\right) .
$$

Proposition 3.7 (homological vanishing by iteration). Assume that $\boldsymbol{q}$ is not a local maximum for $L^{\times n}$. Then, for each integer $p \geq 2$, there exists $\bar{m}=\bar{m}(\boldsymbol{q}, p) \in \mathbb{N}$ that is a nonnegative power of $p$ such that the homomorphism $\psi_{*}^{\times \bar{m}}$ as in (3-11) is the zero one.

The proof is based on a homotopical result that we discuss now. Consider a point $\boldsymbol{q}=\left(q_{0}, \ldots, q_{n-1}\right)$ in the space $\mathrm{G}_{\epsilon}^{\times n}(S)$ and, for each $j \in \mathbb{Z}_{n}$, a sufficiently small neighborhood $U_{j} \subset S$ of $q_{j}$, so that $U:=U_{0} \times \cdots \times U_{n-1}$ is an open neighborhood of $\boldsymbol{q}$ in $\mathrm{G}_{\epsilon}^{\times n}(S)$. For each $c \in \mathbb{R}$, we denote by $U_{>c}$ the set of points $\boldsymbol{q}^{\prime}$ in $U$ such that $L^{\times n}\left(\boldsymbol{q}^{\prime}\right)>c$. Analogously, for each $m \in \mathbb{N}$, we denote by $U_{>c}^{\times m}$ the set of points $\boldsymbol{q}^{\prime \prime}$ in $U^{\times m}$ such that $L^{\times n m}\left(\boldsymbol{q}^{\prime \prime}\right)>c$. Notice that the iteration map $\psi^{\times m}$ send $U_{>c}$ into $U_{>m c}^{\times m}$. Then, for each $j \in \mathbb{N}$, we denote by $\Delta^{j}$ the standard $j$-simplex in $\mathbb{R}^{j}$.

Lemma 3.8 (homotopical vanishing by iteration). Let $b<c$ such that $U_{>c} \neq$ $\varnothing$, and consider the singular simplex $\sigma:\left(\Delta^{j}, \partial \Delta^{j}\right) \rightarrow\left(U_{>b}, U_{>c}\right)$, i.e., $[\sigma] \in$ $\pi_{j}\left(U_{>b}, U_{>c}\right)$. Then, there exists $\bar{m}=\bar{m}(\sigma) \in \mathbb{N}$ and, for each integer $m \geq \bar{m}, a$ homotopy

$$
\sigma^{\bigotimes m}:[0,1] \times\left(\Delta^{j}, \partial \Delta^{j}\right) \rightarrow\left(U_{>m b}^{\times m}, U_{>m c}^{\times m}\right)
$$

such that

(i) $\sigma^{\bigotimes m}(0, \cdot)=\sigma^{\times m}:=\psi^{\times m} \circ \sigma$,

(ii) $\sigma^{\bowtie m}(t, x)=\sigma^{\bowtie m}(0, x)$ for each $x \in \partial \Delta^{j}$,

(iii) $\sigma^{\bigotimes m}\left(1, \Delta^{j}\right) \subset U_{>m c}^{\times m}$.

In particular, $\left[\sigma^{\times m}\right]=0$ in $\pi_{j}\left(U_{>m b}^{\times m}, U_{>m c}^{\times m}\right)$. 
Proof. We begin by explaining the basic construction that will be employed in the proof. Consider a map $\gamma:\left[x_{0}, x_{1}\right] \rightarrow U$, where $\left[x_{0}, x_{1}\right] \subset \mathbb{R}$. For each $m \in \mathbb{N}$ we define a map

$$
\gamma^{\square m}:\left[x_{0}, x_{1}\right] \rightarrow U^{\times m}
$$

in the following way: for each $k \in\{0, \ldots, m-1\}$ and $y \in\left[x_{0}, x_{1}\right]$, denoting $\boldsymbol{q}_{0}=\gamma\left(x_{0}\right), \boldsymbol{q}_{1}=\gamma\left(x_{1}\right)$ and $x=x_{0}+\left(x_{1}-x_{0}\right) \frac{k}{m}+\frac{y-x_{0}}{m}$, we set

$$
\gamma^{\square m}(x):=(\underbrace{\boldsymbol{q}_{1}, \ldots, \boldsymbol{q}_{1}}_{\times k}, \gamma(y), \underbrace{\boldsymbol{q}_{0}, \ldots, \boldsymbol{q}_{0}}_{\times m-k-1}) .
$$

The length of $\gamma^{\square m}(x)$ is bounded from below as

$$
\begin{aligned}
L^{\times n m}\left(\gamma^{\square m}(x)\right) & \geq(k-1) L^{\times n}\left(\boldsymbol{q}_{1}\right)+(m-k-2) L^{\times n}\left(\boldsymbol{q}_{0}\right) \\
& \geq(m-3) \min \left\{L^{\times n}\left(\gamma\left(x_{0}\right)\right), L^{\times n}\left(\gamma\left(x_{1}\right)\right)\right\} .
\end{aligned}
$$

Now, consider the singular simplex $\sigma$ of the statement. We want to decompose its domain $\Delta^{j}$ as a "continuous" family of paths, and then apply the above construction to each path separately. Let $\mathbb{L} \subseteq \mathbb{R}^{j}$ be the axis passing through the origin and the barycenter of $\Delta^{j} \subset \mathbb{R}^{j}$. For each $s \in[0,1]$ we denote by $s \Delta^{j}$ the rescaled $j$-simplex given by $\left\{s z \mid z \in \Delta^{j}\right\}$. For each $z \in s \Delta^{j}$, we denote by $\left[x_{0}(s, z), x_{1}(s, z)\right] \subset s \Delta^{j}$ the maximum segment that contains $z$ and is parallel to L. This notation is summarized, for $j=2$, as follows:

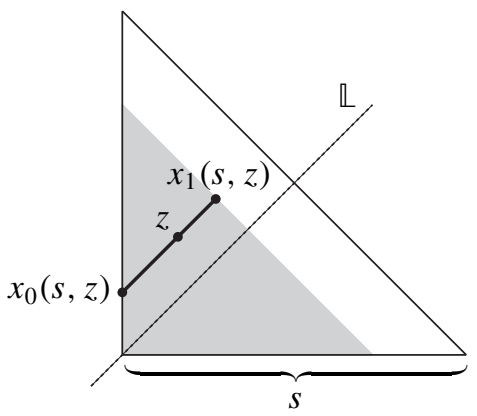

We define the homotopy $\sigma^{\bigotimes m}:[0,1] \times \Delta^{j} \rightarrow U$ by

$$
\sigma^{\bigotimes m}(s, z)= \begin{cases}\left(\left.\sigma\right|_{\left[x_{0}(s, z), x_{1}(s, z)\right]}\right)^{\square m}(z) & \text { if } z \in s \Delta^{j}, \\ \sigma^{\times m}(z) & \text { if } z \notin s \Delta^{j} .\end{cases}
$$

This homotopy clearly satisfies properties (i)-(ii) in the statement. Then, for each $s \in[0,1]$ and $z \in s \Delta^{j}$, by the estimate in (3-13) we have

$$
L^{\times n m}\left(\sigma^{\bigotimes m}(s, z)\right) \geq(m-3) \min \left\{L^{\times n}\left(\sigma\left(x_{0}(s, z)\right)\right), L^{\times n}\left(\sigma\left(x_{1}(s, z)\right)\right)\right\},
$$


while for each $z \in \Delta^{j} \backslash s \Delta^{j}$ we have

$$
L^{\times n m}\left(\sigma^{\bowtie m}(s, z)\right)=L^{\times n m}\left(\sigma^{\times m}(z)\right)=m L^{\times n}(\sigma(z)) .
$$

Choose $\delta>0$ sufficiently small so that $\sigma\left(\Delta^{j}\right) \subset U_{>b+\delta}$ and $\sigma\left(\partial \Delta^{j}\right) \subset U_{>c+\delta}$. For each $(s, z) \in[0,1] \times \Delta^{j}$ we obtain

$$
\begin{aligned}
& L^{\times n m}\left(\sigma^{\bigotimes m}(s, z)\right) \geq(m-3)(b+\delta), \\
& L^{\times n m}\left(\sigma^{\bigotimes m}(1, z)\right) \geq(m-3)(c+\delta) .
\end{aligned}
$$

This implies that, for $m \in \mathbb{N}$ sufficiently big, the homotopy $\sigma^{\bigotimes m}$ has the form (3-12) and satisfies (iii).

Proof of Proposition 3.7. The proof is based on the homotopical vanishing principle of Lemma 3.8, together with the homotopical invariance property of singular homology as stated in [Bangert and Klingenberg 1983, Lemma 1].

Fix the integer $p \geq 2$ of the statement and set

$$
\mathbb{K}_{p}=\left\{p^{n} \mid n \in \mathbb{N} \cup\{0\}\right\} .
$$

Since the local homology group $\mathrm{C}_{*}(\boldsymbol{q})$ is finitely generated (see [Gromoll and Meyer 1969a, Lemma 2]), all we need to prove is that, for each homology class $[\mu] \in \mathrm{C}_{*}(\boldsymbol{q})$ and for some $\bar{m}=\bar{m}([\mu]) \in \mathbb{K}_{p}$, we have

$$
\psi_{*}^{\times \bar{m}}[\mu]=0 \quad \text { in } \mathrm{H}_{*}\left(\mathrm{G}_{\epsilon}^{\times n \bar{m}}(S)_{>\bar{m} b}, \mathrm{G}_{\epsilon}^{\times n \bar{m}}(S)_{>\bar{m} c}\right) .
$$

Let $U$ be the open neighborhood of $\boldsymbol{q}$ introduced in the paragraph preceding Lemma 3.8. By excision, we can assume that the relative cycle $\mu$ representing $[\mu]$ has support contained in $U$. We denote by $\Sigma(\mu)$ the collection of singular simplices in $\mu$ and all their lower-dimensional faces. For each $\sigma: \Delta^{j} \rightarrow U$ contained in $\Sigma(\mu)$ we will define $\bar{m}=\bar{m}(\sigma) \in \mathbb{K}_{p}$ and a homotopy

$$
\sigma^{\bullet \bar{m}}:[0,1] \times \Delta^{j} \rightarrow U_{>\bar{m} b}^{\times \bar{m}}
$$

such that

(i) $\sigma^{\bullet \bar{m}}(0, \cdot)=\sigma^{\times \bar{m}}$,

(ii) $\sigma^{\bullet \bar{m}}\left(1, \Delta^{j}\right) \subset U_{>\bar{m} c}^{\times \bar{m}}$,

(iii) if $\sigma\left(\Delta^{j}\right) \subset U_{>c}$, then $\sigma^{\bullet \bar{m}}(s, \cdot)=\sigma^{\times \bar{m}}$ for each $s \in[0,1]$,

(iv) $\left(\sigma \circ F_{i}\right)^{\bullet \bar{m}}=\sigma^{\bullet \bar{m}}\left(\cdot, F_{i}(\cdot)\right)$ for each $i \in\{0, \ldots, j\}$, where $F_{i}: \Delta^{j-1} \rightarrow \Delta^{j}$ is the standard affine map onto the $i$-th face of $\Delta^{j}$.

For each $m \in \mathbb{K}_{p}$ greater than $\bar{m}$, define a homotopy $\sigma^{\bullet m}:[0,1] \times \Delta^{j} \rightarrow U_{>m b}^{\times m}$ by

$$
\sigma^{\bullet m}:=\psi^{\times m / \bar{m}} \circ \sigma^{\bullet \bar{m}} \text {. }
$$


This homotopy satisfies the analogous properties (i)-(iv) in period $m$. Notice that property (iv) implicitly requires that $\bar{m}\left(\sigma \circ F_{i}\right) \leq \bar{m}(\sigma)$ for each $i \in\{0, \ldots, j\}$.

Now, take a sufficiently big $m \in \mathbb{K}_{p}$ so that $\sigma^{\bullet m}$ is defined for all $\sigma \in \Sigma(\mu)$. Then, by means of the above homotopies, the relative cycle $\mu^{\times m}=\psi^{\times m} \circ \mu$ is homologous to a relative cycle whose support is contained in $U_{>m c}^{\times m} \subset \mathrm{G}_{\epsilon}^{\times m}(S)_{>m c}$. In fact, this latter relative cycle is obtain from $\mu^{\times m}$ by homotoping each singular simplex $\sigma^{\times m} \in \Sigma\left(\mu^{\times m}\right)$ to $\sigma^{\bullet m}(1, \cdot)$ via the homotopy $\sigma^{\bullet m}$. This implies that $\psi_{*}^{\times m}[\mu]=\left[\mu^{\times m}\right]=0$ in $\mathrm{H}_{*}\left(\mathrm{G}_{\epsilon}^{\times n m}(S)_{>m b}, \mathrm{G}_{\epsilon}^{\times n m}(S)_{>m c}\right)$. In order to finish the proof, we only need to build the above homotopies satisfying (i)-(iv). The idea is to apply Lemma 3.8 subsequently to all the faces of the singular simplices in $\Sigma(\mu)$, starting from 0 -dimensional faces and then going up to higher-dimensional ones.

We proceed by induction, starting by assuming that $\mu$ is a 0 -relative cycle. Then, $\Sigma(\mu)$ is simply a finite set of points in $U_{>b}$. Let $\boldsymbol{w}$ be one of these points. If $\boldsymbol{w} \in U_{>c}$ we are already done: we simply set $\bar{m}=\bar{m}(\boldsymbol{w})=1$ and $\boldsymbol{w}^{\boldsymbol{\bullet}}(s)=\boldsymbol{w}$ for each $s \in[0,1]$. In the other case, $\boldsymbol{w} \in U_{>b} \backslash U_{>c}$, we take an arbitrary continuous path $\gamma:[0,1] \rightarrow U$ such that $\gamma(0)=w$ and $\gamma(1) \in U_{>c}$. Such a path exists, since the critical point $\boldsymbol{q}$ of the statement is not a local minimum and therefore, since $L^{\times n}(\boldsymbol{q})=c$, the subset $U_{>c}$ is not empty. Then, consider $\bar{m}=\bar{m}(\gamma) \in \mathbb{N}$ and the associated homotopy

$$
\gamma^{\bigotimes \bar{m}}:[0,1] \times[0,1] \rightarrow U^{\times \bar{m}}
$$

given by Lemma 3.8, such that

$$
\gamma^{\bigotimes \bar{m}}(s, 0)=\boldsymbol{w}^{\times \bar{m}}, \quad \gamma^{\bigotimes \bar{m}}(s, 1)=\gamma(1)^{\times \bar{m}}, \quad \gamma^{\bigotimes \bar{m}}(1, s) \in U_{>\bar{m} b}^{\times \bar{m}}
$$

for each $s \in[0,1]$. Without loss of generality we can assume that $\bar{m} \in \mathbb{K}_{p}$, and we set

$$
\boldsymbol{w}^{\bullet \bar{m}}=\gamma^{\bigotimes \bar{m}}(1, \cdot)
$$

When $\mu$ is a $j$-relative cycle, with $j \geq 1$, we can apply the inductive hypothesis: for every nonnegative integer $j^{\prime} \leq j-1$ and for each $j^{\prime}$-singular simplex $\sigma \in \Sigma(\mu)$ we obtain $\bar{m}=\bar{m}(\sigma) \in \mathbb{K}_{p}$ and a homotopy $\sigma^{\bullet \bar{m}}$ satisfying the above properties (i) (iv). Now, consider a $j$-singular simplex $\sigma \in \Sigma(\mu)$. If $\sigma\left(\Delta^{j}\right) \subset U_{>c}$ we simply set $\bar{m}=\bar{m}(\sigma):=1$ and $\sigma^{\bullet \bar{m}}(s, \cdot):=\sigma$ for each $s \in[0,1]$. Otherwise, if $\sigma\left(\Delta^{j}\right) \not \subset U_{>c}$, we denote by $\bar{m}^{\prime}=\bar{m}^{\prime}(\sigma)$ the maximum of the $\bar{m}(v)$ 's for all the proper faces $v$ of $\sigma$. For each $m \in \mathbb{K}_{p}$ greater or equal than $\bar{m}^{\prime}$, every such $v$ has an associated homotopy $\nu^{\bullet m}$ satisfying the above condition (i)-(iv). For technical reasons, let us assume that $v^{\bullet m}(s, \cdot)=v^{\bullet m}\left(\frac{1}{2}, \cdot\right)$ for each $s \in\left[\frac{1}{2}, 1\right]$. Patching together the homotopies of the proper faces of $\sigma$, we obtain

$$
\sigma^{\bullet m}:\left(\left[0, \frac{1}{2}\right] \times \partial \Delta^{j}\right) \cup\left(\{0\} \times \Delta^{j}\right) \rightarrow U_{>m b}^{\times m},
$$


such that $\sigma^{\bullet m}(0, \cdot)=\sigma^{\times m}$ and $\sigma^{\bullet m}\left(\cdot, F_{i}(\cdot)\right)=\left(\sigma \circ F_{i}\right)^{\bullet m}$ for each $i=0, \ldots, j$. By retracting $\left[0, \frac{1}{2}\right] \times \Delta^{j}$ onto $\left(\left[0, \frac{1}{2}\right] \times \partial \Delta^{j}\right) \cup\left(\{0\} \times \Delta^{j}\right)$ we can extend the homotopy $\sigma^{\bullet m}$, obtaining

$$
\sigma^{\bullet m}:\left[0, \frac{1}{2}\right] \times \Delta^{j} \rightarrow U_{>m b}^{\times m} .
$$

Notice that $\sigma^{\bullet \bar{m}^{\prime}}\left(\frac{1}{2}, \cdot\right)$ is a singular simplex of the form

$$
\sigma^{\bullet \bar{m}^{\prime}}\left(\frac{1}{2}, \cdot\right):\left(\Delta^{j}, \partial \Delta^{j}\right) \rightarrow\left(U_{>\bar{m}^{\prime} b}^{\times \bar{m}^{\prime}}, U_{>\bar{m}^{\prime} c}^{\times \bar{m}^{\prime}}\right) .
$$

We briefly denote this singular simplex by $\tilde{\sigma}$, and consider $\bar{m}^{\prime \prime}=\bar{m}(\tilde{\sigma})$ and the homotopy $\tilde{\sigma}^{\bigotimes \bar{m}^{\prime \prime}}$ given by Lemma 3.8, so that

$$
\begin{aligned}
& \tilde{\sigma}^{\bigotimes \bar{m}^{\prime \prime}}(0, \cdot)=\tilde{\sigma}^{\times \bar{m}^{\prime \prime}}=\sigma^{\bullet \bar{m}^{\prime \prime} \bar{m}^{\prime}}\left(\frac{1}{2}, \cdot\right), \\
& \tilde{\sigma}^{\bigotimes \bar{m}^{\prime \prime}}\left(1, \Delta^{j}\right) \subset U_{>\bar{m}^{\prime} \bar{m}^{\prime \prime} c}^{\times \bar{m}^{\prime} \bar{m}^{\prime \prime}} .
\end{aligned}
$$

Then, we set $\bar{m}=\bar{m}(\sigma):=\bar{m}^{\prime} \bar{m}^{\prime \prime}$ and we extend the homotopy in (3-14) to [0,1] $\times$ $\Delta^{j}$ by

$$
\sigma^{\bullet \bar{m}}(s, \cdot):=\tilde{\sigma}^{\bigotimes \bar{m}^{\prime \prime}}(2 s-1, \cdot) \quad \text { for all } s \in\left[\frac{1}{2}, 1\right]
$$

\section{Proof of the main result}

In this section we carry out the proof of Theorem 1.1, stated in the introduction. We adopt the notation of Section 2, so that our billiard table is the strictly convex compact subset $U_{S}$ enclosed by a smooth hypersurface $S \subset \mathbb{R}^{N+1}$, with $N \geq 2$. The proof of Theorem 1.1 will be by contradiction: let us fix, once for all, a prime number $p \in \mathbb{N}$ and let us assume that the following two conditions hold:

(F1) For each $n \in \mathbb{N}$, there are only finitely many periodic billiard trajectories bouncing $p^{n}$ times.

(F2) There are only finitely many periodic billiard trajectories $\gamma_{1}, \ldots, \gamma_{r}$ satisfying properties (i) and (ii) in the statement.

Define $\mathbb{K}_{p}:=\left\{p^{n} \mid n \in \mathbb{N} \cup\{0\}\right\}$. For each $\alpha \in\{1, \ldots, r\}$, let us denote by $\tilde{\boldsymbol{q}}_{\alpha}=\left(\tilde{q}_{\alpha, 1}, \ldots, \tilde{q}_{\alpha, n_{\alpha}}\right) \in \mathrm{G}^{\times n_{\alpha}}(S)$ the sequence of bounce points of $\gamma_{\alpha}$. Let $n \in \mathbb{K}_{p}$ be the maximum of the $n_{\alpha}$ 's, and let us set

$$
\boldsymbol{q}_{\alpha}:=\tilde{\boldsymbol{q}}_{\alpha}^{\times n / n_{\alpha}} \in \mathrm{G}^{\times n}(S) \text { for all } \alpha \in\{1, \ldots, r\} .
$$

Remark 4.1. For each critical point $q$ of the length functional $L^{\times n}$, the function $m \mapsto \operatorname{coind}\left(\boldsymbol{q}^{\times m}\right)$, where $m \in \mathbb{K}_{p}$, is monotone increasing (although not strictly). In fact, the differential of the iteration map $\psi^{\times m}$ at $\boldsymbol{q}$ maps (injectively) any positive eigenspace of hess $L^{\times n}(\boldsymbol{q})$ to a positive eigenspace of hess $L^{\times n m}\left(\boldsymbol{q}^{\times m}\right)$. This implies that, by Assumption (F2), for all $m \in \mathbb{K}_{p}$ the set of critical points of $L^{\times n m}$ 
with Morse coindex less or equal than $N$ is given by the $\mathbb{D}_{n m}$-orbits of those $\boldsymbol{q}_{\alpha}^{\times m}$ 's such that $\operatorname{coind}\left(\boldsymbol{q}_{\alpha}^{\times m}\right) \leq N$.

As a first step in our proof, let us establish the following claim. We refer the reader to pages 193-194 for the definition and properties of local homology groups. From now on, all the homology groups are assumed to have coefficients in $\mathbb{Z}_{2}$.

Claim. There exists $\boldsymbol{q} \in\left\{\boldsymbol{q}_{1}, \ldots, \boldsymbol{q}_{r}\right\}$ with $\overline{\operatorname{coind}}(\boldsymbol{q})=0$ and an infinite subset $\mathbb{K}_{p}^{\prime} \subset \mathbb{K}_{p}$ such that, for each $m \in \mathbb{K}_{p}^{\prime}$, the local homology group $\mathrm{C}_{N-1}\left(\boldsymbol{q}^{\times m}\right)$ is nontrivial.

Proof. For each $m \in \mathbb{K}_{p}$, fix a sufficiently small $\epsilon=\epsilon(m)>0$ and consider the space $\mathrm{G}_{\epsilon}^{\times n m}(S)$ introduced in Section 2. By Proposition 2.1(iii), this space contains all the critical points of $L^{\times n m}$. Proposition 2.1(ii) implies that the inclusion $\mathrm{G}_{\epsilon}^{\times n m}(S) \hookrightarrow \mathrm{G}^{\times n m}(S)$ induces an isomorphism in homology, and therefore by (2-2) we infer that

$$
\mathrm{H}_{N-1}\left(\mathrm{G}_{\epsilon}^{\times n m}(S)\right) \neq 0 .
$$

By Proposition 2.1(i,iv), the functional $-L^{\times n m}: \mathrm{G}_{\epsilon}^{\times n m}(S) \rightarrow \mathbb{R}$ satisfies the "general boundary conditions" for Morse theory (see [Chang 1993, Section 6.1]), and we have the Morse inequality

$$
\operatorname{dim} \mathrm{H}_{k}\left(\mathrm{G}_{\epsilon}^{\times n m}(S)\right) \leq \sum_{\boldsymbol{q}^{\prime}} \operatorname{dim} \mathrm{C}_{k}\left(\boldsymbol{q}^{\prime}\right)
$$

where the sum in the right hand side runs over all the critical points $\boldsymbol{q}^{\prime}$ of $L^{\times n m}$. Notice that only those $\boldsymbol{q}^{\prime}$ such that

$$
\operatorname{coind}\left(\boldsymbol{q}^{\prime}\right) \leq k \leq \operatorname{coind}\left(\boldsymbol{q}^{\prime}\right)+\operatorname{nul}\left(\boldsymbol{q}^{\prime}\right)
$$

may possibly give a nonzero contribution. Now, choosing $k=N-1$, by Remark 4.1 the elements in the $\mathbb{D}_{n m}$-orbit of the $\boldsymbol{q}_{\alpha}^{\times m}$ 's are the only critical points $\boldsymbol{q}^{\prime}$ of $L^{\times n m}$ that may satisfy (4-3). Hence, by (4-1) and by the Morse inequality (4-2) we infer

$$
0 \neq \sum_{\alpha=1}^{r} \operatorname{dim} C_{N-1}\left(\mathbb{D}_{n m} \cdot\left\{\boldsymbol{q}_{\alpha}^{\times m}\right\}\right) .
$$

Now, if all the $\boldsymbol{q}_{\alpha}$ 's had nonzero mean Morse coindex $\overline{\operatorname{coind}}\left(\boldsymbol{q}_{\alpha}\right)$, by the iteration inequality in Proposition 3.1(ii) we would have $\operatorname{coind}\left(\boldsymbol{q}_{\alpha}^{\times m}\right)>N-1$, providing $m \in \mathbb{K}_{p}$ is big enough. However, this would imply that $\mathrm{C}_{N-1}\left(\mathbb{D}_{n m} \cdot\left\{\boldsymbol{q}_{\alpha}^{\times m}\right\}\right)=0$ for each $\alpha \in\{1, \ldots, r\}$, contradicting (4-4). Hence, some of the $\boldsymbol{q}_{\alpha}$ 's, say $\boldsymbol{q}_{1}, \ldots, \boldsymbol{q}_{s}$ where $s \leq r$, satisfy $\overline{\operatorname{coind}}\left(\boldsymbol{q}_{\alpha}\right)=0$. Up to choosing $m$ big enough the inequality 
in (4-4) reduces to

$$
0 \neq \sum_{\alpha=1}^{s} \operatorname{dim} C_{N-1}\left(\mathbb{D}_{n m} \cdot\left\{\boldsymbol{q}_{\alpha}^{\times m}\right\}\right) .
$$

This implies that at least one $\boldsymbol{q}$ among $\boldsymbol{q}_{1}, \ldots, \boldsymbol{q}_{s}$ satisfies $\mathrm{C}_{N-1}\left(\mathbb{D}_{n m} \cdot\left\{\boldsymbol{q}^{\times m}\right\}\right) \neq 0$, and thus $\mathrm{C}_{N-1}\left(\boldsymbol{q}^{\times m}\right) \neq 0$ as well, for infinitely many $m \in \mathbb{K}_{p}$.

Now, by Proposition 3.1, the indices coind $\left(\boldsymbol{q}^{\times m}\right)$ and $\operatorname{nul}\left(\boldsymbol{q}^{\times m}\right)$ are uniformly bounded for all $m \in \mathbb{K}_{p}^{\prime}$, and hence we can choose an infinite subset $\mathbb{K}_{p}^{\prime \prime} \subset \mathbb{K}_{p}^{\prime}$ such that $\operatorname{coind}\left(\boldsymbol{q}^{\times m}\right)$ and $\operatorname{nul}\left(\boldsymbol{q}^{\times m}\right)$ are constant in $m \in \mathbb{K}_{p}^{\prime \prime}$. Without loss of generality, let us assume that 1 belongs to $\mathbb{K}_{p}^{\prime \prime}$ (equivalently, set $m:=\min \mathbb{K}_{p}^{\prime \prime}$ and rename $\boldsymbol{q}$ to be $\boldsymbol{q}^{\times m}$ and $\mathbb{K}_{p}^{\prime \prime}$ to be $m^{-1} \mathbb{K}_{p}^{\prime \prime}$ ).

We set $c:=L^{\times n}(\boldsymbol{q})$ and we fix an arbitrary real number $b<c$ such that none of the $\boldsymbol{q}_{\alpha}$ 's has critical value in the open interval $(b, c)$, i.e., $L^{\times n}\left(\boldsymbol{q}_{\alpha}\right) \notin(b, c)$ for all $\alpha \in\{1, \ldots, r\}$. Now, for each $m \in \mathbb{K}_{p}^{\prime \prime}$, let us choose $\epsilon=\epsilon(m)>0$ small enough so that $\mathrm{G}_{\epsilon}^{\times n}(S)$ and $\mathrm{G}_{\epsilon}^{\times n m}(S)$ satisfy assertions (i)-(iv) of Proposition 2.1. Then, let us consider the iteration map restricted as a map of pairs of the form

$$
\psi^{\times m}:\left(\mathrm{G}_{\epsilon}^{\times n}(S)_{>c} \cup\{\boldsymbol{q}\}, \mathrm{G}_{\epsilon}^{\times n}(S)_{>c}\right) \hookrightarrow\left(\mathrm{G}_{\epsilon}^{\times n m}(S)_{>m b}, \mathrm{G}_{\epsilon}^{\times n m}(S)_{>m c}\right) .
$$

Claim. For each $m \in \mathbb{K}_{p}^{\prime \prime}$, the iteration map in (4-5) is injective in degree $(N-1)$ homology, i.e.,

$$
\psi_{*}^{\times m}: \mathrm{C}_{N-1}(\boldsymbol{q}) \hookrightarrow \mathrm{H}_{N-1}\left(\mathrm{G}_{\epsilon}^{\times n m}(S)_{>m b}, \mathrm{G}_{\epsilon}^{\times n m}(S)_{>m c}\right) \quad \text { for all } m \in \mathbb{K}_{p}^{\prime \prime}
$$

Proof. Consider an arbitrary $m \in \mathbb{K}_{p}^{\prime \prime}$. By Assumption (F1), we can choose $b^{\prime} \in$ $(m b, m c)$ sufficiently close to $m c$ such that the only critical value of $L^{\times n m}$ in the interval $\left(b^{\prime}, m c\right.$ ] is $m c$. By Morse theory (see [Chang 1993, Theorem 4.2], for example), the inclusion

$$
\left(\mathrm{G}_{\epsilon}^{\times n m}(S)_{>c} \cup\left\{\boldsymbol{q}^{\times m}\right\}, \mathrm{G}_{\epsilon}^{\times n m}(S)_{>c}\right) \hookrightarrow\left(\mathrm{G}_{\epsilon}^{\times n m}(S)_{>b^{\prime}}, \mathrm{G}_{\epsilon}^{\times n m}(S)_{>m c}\right)
$$

is injective in homology, namely it induces the monomorphism

$$
\mathrm{C}_{*}\left(\boldsymbol{q}^{\times m}\right) \hookrightarrow \mathrm{H}_{*}\left(\mathrm{G}_{\epsilon}^{\times n m}(S)_{>b^{\prime}}, \mathrm{G}_{\epsilon}^{\times n m}(S)_{>m c}\right) .
$$

Now, let us examine the long exact sequence of the triple

$$
\left(\mathrm{G}_{\epsilon}^{\times n m}(S)_{>m b}, \mathrm{G}_{\epsilon}^{\times n m}(S)_{>b^{\prime}}, \mathrm{G}_{\epsilon}^{\times n m}(S)_{>m c}\right),
$$


which we can write as the following exact triangle:

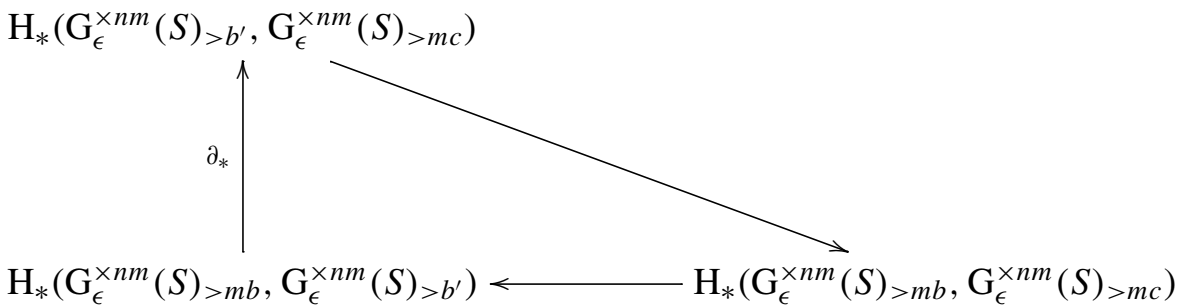

Here, $\partial_{*}$ is the boundary homomorphism which lower the grade $*$ by one, and the other homomorphisms are simply induced by inclusions. Now, by our choice of $b$ and $b^{\prime}$, none of the $\boldsymbol{q}_{\alpha}^{\times m}$ 's has critical value inside the interval $\left(m b, b^{\prime}\right]$ and, by Assumption (F2), all the critical values of $L^{\times n m}$ inside $\left(m b, b^{\prime}\right]$ correspond to critical points that either are local maxima or have Morse coindex greater than $N$. This, together with the Morse inequalities, implies that the group

$$
\mathrm{H}_{j}\left(\mathrm{G}_{\epsilon}^{\times n m}(S)_{>m b}, \mathrm{G}_{\epsilon}^{\times n m}(S)_{>b^{\prime}}\right)
$$

is trivial in degree $j=N-1$ and $j=N$. Hence, the above exact triangle implies that the diagonal homomorphism is an isomorphism in degree $N-1$, i.e., the inclusion induces the homology isomorphism

$$
\mathrm{H}_{N-1}\left(\mathrm{G}_{\epsilon}^{\times n m}(S)_{>b^{\prime}}, \mathrm{G}_{\epsilon}^{\times n m}(S)_{>m c}\right) \stackrel{\cong}{\longrightarrow} \mathrm{H}_{N-1}\left(\mathrm{G}_{\epsilon}^{\times n m}(S)_{>m b}, \mathrm{G}_{\epsilon}^{\times n m}(S)_{>m c}\right) .
$$

This isomorphism and the monomorphism in (4-6) fit in the following commutative diagram, where all the homomorphisms are induced by inclusions:

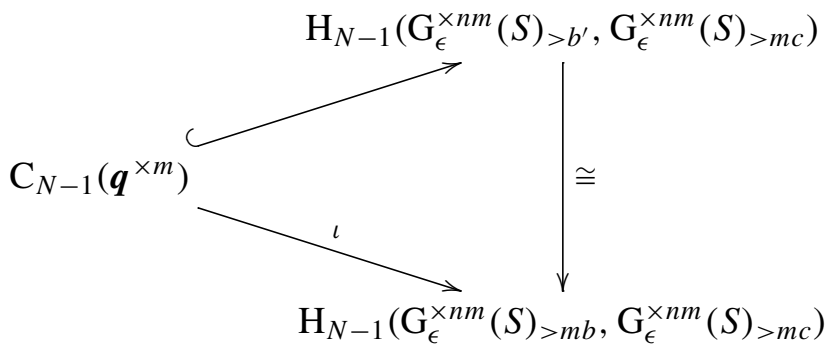

This forces the inclusion-induced homomorphism $\iota$ to be injective:

$$
\iota: \mathrm{C}_{N-1}\left(\boldsymbol{q}^{\times m}\right) \hookrightarrow \mathrm{H}_{N-1}\left(\mathrm{G}_{\epsilon}^{\times n m}(S)_{>b^{\prime}}, \mathrm{G}_{\epsilon}^{\times n m}(S)_{>m c}\right) .
$$

Now, by Proposition 3.5, the iteration map induces the homology isomorphism

$$
\psi_{*}^{\times m}: \mathrm{C}_{*}(\boldsymbol{q}) \stackrel{\cong}{\longrightarrow} \mathrm{C}_{*}\left(\boldsymbol{q}^{\times m}\right) .
$$

By composing the monomorphism $\iota$ with the isomorphism $\psi_{*}^{\times m}$ we obtain the claim. 
For each $m \in \mathbb{K}_{p}^{\prime \prime}$, the critical point $\boldsymbol{q}^{\times m}$ of $L^{\times n m}$ is not a local maximum. In fact, $\mathrm{C}_{N-1}\left(\boldsymbol{q}^{\times m}\right)$ is nontrivial whereas the local homology groups of a local maximum are nontrivial only in degree zero (we recall that $N \geq 2$ ). Therefore, by the homological vanishing principle in Proposition 3.7, the homomorphism

$$
\psi_{*}^{\times m}: \mathrm{C}_{*}(\boldsymbol{q}) \rightarrow \mathrm{H}_{*}\left(\mathrm{G}_{\epsilon}^{\times n m}(S)_{>m b}, \mathrm{G}_{\epsilon}^{\times n m}(S)_{>m c}\right)
$$

is zero provided $m \in \mathbb{K}_{p}^{\prime \prime}$ is sufficiently big. This contradicts the claim, and concludes the proof of Theorem 1.1.

\section{Acknowledgments}

The author thanks Alberto Abbondandolo, Sergei Tabachnikov and the anonymous referee for encouraging and for useful remarks on a preliminary version of the paper. This research has been supported by the Max Planck Institute for Mathematics in the Sciences (Leipzig, Germany) and by the ANR project "KAM faible".

\section{References}

[Arnold 1969] V. I. Arnold, "The cohomology ring of the group of dyed braids", Mat. Zametki 5 (1969), 227-231. MR 39 \#3529

[Babenko 1990] I. K. Babenko, "Periodic trajectories of three-dimensional Birkhoff billiards", Mat. Sb. 181:9 (1990), 1155-1169. In Russian; translated in Math. USSR Sb. 71:1 (1992), 1-13. MR 91m:58128 Zbl 0713.58007

[Bangert 1980] V. Bangert, "Closed geodesics on complete surfaces", Math. Ann. 251:1 (1980), 83-96. MR 81k:58027 Zbl 0422.53024

[Bangert 1988] V. Bangert, "Mather sets for twist maps and geodesics on tori”, pp. 1-56 in Dynamics reported, vol. 1, Dynam. Report. Ser. Dynam. Systems Appl. 1, Wiley, Chichester, 1988. MR 90a:58145 Zbl 0664.53021

[Bangert and Klingenberg 1983] V. Bangert and W. Klingenberg, "Homology generated by iterated closed geodesics”, Topology 22:4 (1983), 379-388. MR 715246 (85b:58031

[Birkhoff 1927] G. D. Birkhoff, "On the periodic motions of dynamical systems", Acta Math. 50:1 (1927), 359-379. MR 1555257

[Bott 1956] R. Bott, "On the iteration of closed geodesics and the Sturm intersection theory", Comm. Pure Appl. Math. 9 (1956), 171-206. MR 19,859f Zbl 0074.17202

[Chang 1993] K.-c. Chang, Infinite-dimensional Morse theory and multiple solution problems, Prog. in Nonlinear Diff. Eq. Appl. 6, Birkhäuser, Boston, 1993. MR 94e:58023 Zbl 0779.58005

[Cohen 1988] F. R. Cohen, "Artin's braid groups, classical homotopy theory, and sundry other curiosities", pp. 167-206 in Braids (Santa Cruz, CA, 1986), Contemp. Math. 78, Amer. Math. Soc., Providence, RI, 1988. MR 90a:20077

[Cohen 1995] F. R. Cohen, "On configuration spaces, their homology, and Lie algebras", J. Pure Appl. Algebra 100:1-3 (1995), 19-42. MR 1344842 (96d:55005

[Croft and Swinnerton-Dyer 1963] H. T. Croft and H. P. F. Swinnerton-Dyer, "On the Steinhaus billiard table problem”, Proc. Cambridge Philos. Soc. 59 (1963), 37-41. MR 0145394 (26 \#2925 
[Farber 2002] M. Farber, “Topology of billiard problems, I, II”, Duke Math. J. 115:3 (2002), 559585, 587-621. MR 2004a:55020

[Farber and Tabachnikov 2002a] M. Farber and S. Tabachnikov, "Periodic trajectories in 3-dimensional convex billiards", Manuscripta Math. 108:4 (2002), 431-437. MR 1923531 (2003h:37106

[Farber and Tabachnikov 2002b] M. Farber and S. Tabachnikov, "Topology of cyclic configuration spaces and periodic trajectories of multi-dimensional billiards", Topology 41:3 (2002), 553-589. MR 2004a:55021

[Gromoll and Meyer 1969a] D. Gromoll and W. Meyer, "On differentiable functions with isolated critical points", Topology 8 (1969), 361-369. MR 39 \#7633 Zbl 0212.28903

[Gromoll and Meyer 1969b] D. Gromoll and W. Meyer, "Periodic geodesics on compact riemannian manifolds", J. Differential Geometry 3 (1969), 493-510. MR 41 \#9143 Zbl 0203.54401

[Karasev 2009] R. N. Karasev, "Periodic billiard trajectories in smooth convex bodies", Geom. Funct. Anal. 19:2 (2009), 423-428. MR 2010m:37055 Zbl 1184.37031

[Kozlov and Treshchëv 1991] V. V. Kozlov and D. V. Treshchëv, Billiards: a genetic introduction to the dynamics of systems with impacts, Translations of Mathematical Monographs 89, American Mathematical Society, Providence, RI, 1991.

[Liu and Long 1998] C. Liu and Y. Long, "An optimal increasing estimate of the iterated Maslovtype indices”, Chinese Sci. Bull. 43:13 (1998), 1063-1066. MR 2000a:53142 Zbl 1002.53055

[Liu and Long 2000] C.-g. Liu and Y. Long, "Iteration inequalities of the Maslov-type index theory with applications”, J. Differential Eq. 165:2 (2000), 355-376. MR 2001k:53153 Zbl 0961.37017

[Long 2000] Y. Long, "Multiple periodic points of the Poincaré map of Lagrangian systems on tori", Math. Z. 233:3 (2000), 443-470. MR 2001a:37093 Zbl 0984.37074

[Long 2002] Y. Long, Index theory for symplectic paths with applications, Progress in Mathematics 207, Birkhäuser, Basel, 2002. MR 2003d:37091 Zbl 1012.37012

[Lu 2009] G. Lu, "The Conley conjecture for Hamiltonian systems on the cotangent bundle and its analogue for Lagrangian systems”, J. Funct. Anal. 256:9 (2009), 2967-3034. MR 2010f:37113 Zbl 1184.53080

[Mazzucchelli 2011] M. Mazzucchelli, “The Lagrangian Conley conjecture”, Comment. Math. Helv. 86:1 (2011), 189-246. MR 2745280 Zbl 1209.37067

Received October 28, 2010. Revised January 19, 2011.

\author{
MARCo MAZZUCCHELli \\ DEPARTMENT OF MATHEMATICS \\ PENN STATE UNIVERSITY \\ UNIVERSITY PARK, PA 16802 \\ UNITED STATES \\ mazzucchel@math.psu.edu \\ http://www.math.psu.edu/mazzucchel
}




\title{
PACIFIC JOURNAL OF MATHEMATICS
}

\author{
http://www.pjmath.org \\ Founded in 1951 by
}

E. F. Beckenbach (1906-1982) and F. Wolf (1904-1989)

\section{EDITORS}

V. S. Varadarajan (Managing Editor)

Department of Mathematics

University of California

Los Angeles, CA 90095-1555

pacific@math.ucla.edu

Vyjayanthi Chari

Department of Mathematics

University of California

Riverside, CA 92521-0135

chari@math.ucr.edu

\section{Robert Finn}

Department of Mathematics Stanford University

Stanford, CA 94305-2125

finn@math.stanford.edu

Kefeng Liu

Department of Mathematics

University of California

Los Angeles, CA 90095-1555

liu@math.ucla.edu
Darren Long

Department of Mathematics

University of California

Santa Barbara, CA 93106-3080

long@math.ucsb.edu

Jiang-Hua Lu

Department of Mathematics

The University of Hong Kong

Pokfulam Rd., Hong Kong jhlu@maths.hku.hk

Alexander Merkurjev

Department of Mathematics University of California

Los Angeles, CA 90095-1555 merkurev@math.ucla.edu
Sorin Popa

Department of Mathematics

University of California

Los Angeles, CA 90095-1555

popa@math.ucla.edu

Jie Qing

Department of Mathematics

University of California

Santa Cruz, CA 95064

qing@ cats.ucsc.edu

Jonathan Rogawski

Department of Mathematics

University of California

Los Angeles, CA 90095-1555

jonr@math.ucla.edu

\section{PRODUCTION}

pacific@math.berkeley.edu

Silvio Levy, Scientific Editor Matthew Cargo, Senior Production Editor

ACADEMIA SINICA, TAIPEI

CALIFORNIA INST. OF TECHNOLOGY

INST. DE MATEMÁTICA PURA E APLICADA

KEIO UNIVERSITY

MATH. SCIENCES RESEARCH INSTITUTE

NEW MEXICO STATE UNIV.

OREGON STATE UNIV.

\section{SUPPORTING INSTITUTIONS}

STANFORD UNIVERSITY
UNIV. OF BRITISH COLUMBIA
UNIV. OF CALIFORNIA, BERKELEY
UNIV. OF CALIFORNIA, DAVIS
UNIV. OF CALIFORNIA, LOS ANGELES
UNIV. OF CALIFORNIA, RIVERSIDE
UNIV. OF CALIFORNIA, SAN DIEGO
UNIV. OF CALIF., SANTA BARBARA

UNIV. OF CALIF., SANTA CRUZ

UNIV. OF MONTANA

UNIV. OF OREGON

UNIV. OF SOUTHERN CALIFORNIA

UNIV. OF UTAH

UNIV. OF WASHINGTON

WASHINGTON STATE UNIVERSITY

These supporting institutions contribute to the cost of publication of this Journal, but they are not owners or publishers and have no responsibility for its contents or policies.

See inside back cover or www.pjmath.org for submission instructions.

The subscription price for 2011 is US \$420/year for the electronic version, and \$485/year for print and electronic.

Subscriptions, requests for back issues from the last three years and changes of subscribers address should be sent to Pacific Journal of Mathematics, P.O. Box 4163, Berkeley, CA 94704-0163, U.S.A. Prior back issues are obtainable from Periodicals Service Company, 11 Main Street, Germantown, NY 12526-5635. The Pacific Journal of Mathematics is indexed by Mathematical Reviews, Zentralblatt MATH, PASCAL CNRS Index, Referativnyi Zhurnal, Current Mathematical Publications and the Science Citation Index.

The Pacific Journal of Mathematics (ISSN 0030-8730) at the University of California, c/o Department of Mathematics, 969 Evans Hall, Berkeley, CA 94720-3840, is published monthly except July and August. Periodical rate postage paid at Berkeley, CA 94704, and additional mailing offices. POSTMASTER: send address changes to Pacific Journal of Mathematics, P.O. Box 4163, Berkeley, CA 94704-0163.

PJM peer review and production are managed by EditFLOW ${ }^{\mathrm{TM}}$ from Mathematical Sciences Publishers.

PUBLISHED BY PACIFIC JOURNAL OF MATHEMATICS

at the University of California, Berkeley 94720-3840

A NON-PROFIT CORPORATION

Typeset in IATEX

Copyright $(2011$ by Pacific Journal of Mathematics 


\section{PACIFIC JOURNAL OF MATHEMATICS}

Volume $252 \quad$ No. $1 \quad$ July 2011

Some dynamic Wirtinger-type inequalities and their applications

RaVi P. Agarwal, Martin Bohner, Donal O'REgAn and SAMIR H.

SAKER

Splitting criteria for vector bundles on higher-dimensional varieties

PARSA BAKHTARY

Average Mahler's measure and $L_{p}$ norms of unimodular polynomials

KWOK-KWOng STEPHen CHOI and Michael J. MossinghofF

Tate resolutions and Weyman complexes

David A. CoX and EVgeny Materov

On pointed Hopf algebras over dihedral groups

FERNANDO FANTINO and GASTON ANDRÉS GARCIA

Integral topological quantum field theory for a one-holed torus

PATRicK M. Gilmer and Gregor Masbaum

Knot 4-genus and the rank of classes in $\boldsymbol{W}(\mathbb{Q}(t))$

CHARLES LIVINGSTON

Roots of Toeplitz operators on the Bergman space

ISSAM LOUHICHI and NAGISETTY V. RAO

Uniqueness of the foliation of constant mean curvature spheres in asymptotically 145 flat 3-manifolds

SHIGUANG MA

On the multiplicity of non-iterated periodic billiard trajectories

MARCo MAZZUCCHELLI

A remark on Einstein warped products

MiCHELE RIMOLDI

Exceptional Dehn surgery on large arborescent knots

YING-QING WU

Harnack estimates for the linear heat equation under the Ricci flow 